\title{
Aerodynamic performance of twin-box decks: a parametric study on gap width effects based on validated 2D URANS simulations
}

\author{
A. J. Álvarez ${ }^{\mathrm{a}}$, F. Nieto ${ }^{\mathrm{a}}$, K.C.S. Kwok ${ }^{\mathrm{b}} \&$ S. Hernández ${ }^{\mathrm{a}}$. \\ a) School of Civil Engineering, University of La Coruña, Campus de Elviña, La Coruña, 15071, Spain. \\ b) School of Civil Engineering, The University of Sydney, Shepherd St, Darlington, NSW 2006, Australia.
}

\begin{abstract}
2D URANS simulations are conducted aiming to study the aerodynamic performance under smooth flow of twin-box decks depending on the gap distance between girders. The Stonecutters Bridge is taken as the reference geometry. In this parametric study, 14 gap to depth ratios in the range $0 \leq G / D \leq 9.70$ are investigated, and for each geometry, 11 angles of attack in the range $-10^{\circ} \leq \alpha \leq 10^{\circ}$ are considered. Specific goals of this research have been: identification of the fundamental flow features, study of mean and fluctuating pressure coefficients distributions, identification of the vortex shedding mechanisms and general aerodynamic characterisation based on force coefficients. The numerical results provided herein are validated with wind tunnel data previously reported in the literature, finding a good agreement. A critical gap to depth ratio at $G / D=2.35$, in terms of aerodynamic response, was identified, which is consistent with the value reported in the literature for a different bridge based on wind tunnel tests. The obtained set of data provide a general picture of the expected aerodynamic performance of a twin-box deck depending on the gap distance and could be of great value at the early design stage of long-span cable-supported bridges.
\end{abstract}

Keywords: 2D URANS, twin box, vortex shedding, gap to depth ratio, force coefficients, Strouhal number, pressure coefficient distribution.

\section{Introduction}

The use of multi-box decks is a feasible alternative for increasing the span length of cable-supported bridges once the limits in the aeroelastic performance of single box decks have been reached. These ultra-long cablesupported bridges are particularly flexible and therefore they are prone to wind action excitation. While multi-box decks have proven to be remarkably efficient in terms of flutter response, they are susceptible to vortex-induced vibration (VIV). Some examples of bridges that have adopted a twin-box deck arrangement are the Yi Sun-sin suspension bridge, in South Korea, the Xihoumen suspension bridge, in China, and the Stonecutters cable-stayed bridge, also located in China.

The aforementioned examples of bridges, that have been designed and built in recent years, have been extensively studied considering their performance under a wide range of aerodynamic and aeroelastic problems. It has been found that the distance between girders plays a significant role in the aerodynamic and aeroelastic responses of twin-box deck bridges. Some relevant references in the frame of the current research are summarized next. The Stonecutters bridge was the first landmark bridge built using this twin-box arrangement. The VIV problem, as well as the Reynolds number dependency of the wind induced response, was extensively studied by means of wind tunnel tests (Larose et al., 2003; Larsen et al., 2005; Larsen et al., 2008; Hui et al., 2008). Furthermore the effect of the gap width in the aerodynamic and aeroelastic responses was addressed in several publications (Qin et al., 2007; Kwok et al., 2012). Another structure that has been also studied in depth is the Xihoumen suspension bridge. Li, Laima and co-workers studied the vortex shedding response and VIV by means of wind tunnel experiments and CFD simulations (Laima et al., 2013); while Reynolds number effects were addressed in another work reported by Li et al. (2014). The impact caused by different gap distances between girders has been investigated in Chen et al. (2014) and in Laima et al. (2015), finding very different flow features depending on the gap width. A simplified section model of the Xihoumen Bridge was used to study the impact of the gap distance in aeroelastic stability (Yang et al., 2015). Furthermore, Reynolds number sensitivity of the aerodynamic response of the Yi Sun-sin Bridge was reported in Lee et al. (2014). The relatively ample bibliography on the subject has allowed identifying three regions in terms of aerodynamic performance 
and flow topology, depending on the gap distance between boxes at $0^{\circ}$ angle of attack. For small gaps, the short distance between boxes prevents the formation and detachment of vortices from the leeward side of the upwind deck, and a weak vortex shedding takes place behind the downwind box. For moderate gaps, a strong von Karman vortex street appears in the wake of the the upwind box, and these vortices impinge upon the downwind box inducing the flow separation at the windward corners of the downwind box. Finally, for large gaps, vortices are freely shed from each box, without mutual interaction.

The references that in recent years have addressed the aerodynamic phenomena in twin box decks by means of wind tunnel tests, and the very brief description of the influence of the gap distance in the flow response that has been introduced in the above paragraph, show the complexity of the involved phenomena, and the difficulties for tackling this problem by means of a numerical approach. In fact, numerical simulations aiming to simulate the aerodynamic response of multi-box decks are scarce, and in some cases the agreement with experimental data could be qualified as poor. An early application was conducted by Nieto and co-workers (Nieto et al., 2008) for the Messina Bridge using the $k-\omega$ SST turbulence model, and another for the Stonecutters bridge (Nieto et al., 2010) using both the realizable $k-\epsilon$ and the $k-\omega$ SST turbulence models. More recent CFD applications can be found in Laima et al. (2013) where 3D Large Eddy Simulations (LES) are used in the frame of the so-called hybrid method aiming to analyse the flow characteristics around a twinbox deck undergoing VIV. Another application consists in the simulation of the aerodynamic response of the multi-box Megane Bridge using Unsteady Reynolds Averaged Navier Stokes (URANS) and LES approaches (Dragomirescu et al., 2016). In Patruno (2015) the force coefficients and the flutter derivatives of different deck cross-sections are obtained by means of CFD simulations using the $k-\omega$ SST turbulence model. Among the considered geometries, the twin-box arrangement of the Gibraltar Bridge proposal is studied. Focusing on the force coefficients, the author has found a fair agreement with experimental data for the drag and lift coefficients, obtaining a poorer agreement for the moment coefficient. A relevant publication in the frame of the research that is going to be reported next is the numerical study on the aerodynamic response of the Xihoumen Bridge deck considering different gaps at $0^{\circ}$ angle of attack (de Miranda et al., 2015). In the latest reference, URANS and LES simulations were conducted aiming to reproduce the experimental results in Chen et al. (2014), where different gap ratios were considered. The URANS simulation were conducted using the $k-\omega$ SST turbulence model. The authors concluded that URANS simulations might be suitable for very small gaps, especially when steady state is reached; nevertheless, URANS did not provide accurate results for intermediate gaps, nor for large gap distances. In the same manner, the transition in the flow topology between small and large gap ratios could not be accurately predicted in the aforementioned work using CFD tools. Finally, LES simulations showed a higher accuracy, since they were able to effectively simulate time-averaged quantities, while pressure fluctuations were qualitatively reproduced. In summary, the results in de Miranda et al. (2015) point towards the lack of reliability of 2D URANS simulations to properly simulate the aerodynamic response of twin-box decks for large gaps. However the relatively small number of elements in the computational grids adopted in that work (55000 cells) opens the door to consider if higher density meshes, in combination with verification studies could improve the ability of 2D URANS models to predict the aerodynamic behaviour of twin-box decks.

The fundamental goal of this work is to explore the ability of 2D URANS CFD simulations to obtain reliable information about the aerodynamic behaviour of twin-box decks in terms of the gap distance between girders ( $G / D$ is the so-called gap ratio in the following; $G$ is the gap distance and $D$ is the depth of the deck crosssection; see figure 1) at different angles of attack. The outputs of interest are the ones that allow characterising the flow around the bridge deck: mean and standard deviation distributions of the pressure coefficient around the boxes, force coefficients and Strouhal number. Furthermore, the flow visualization possibilities, which are characteristic of the numerical approaches to fluid mechanics problems, would shed some light on the flow features for the different gap distances, particularly for the intermediate range, explaining the Region 1 and Region 2 behaviours described later in the manuscript. Using a computational approach, it is also possible to obtain information that is very difficult to get from wind tunnel tests such as the phase lag between the lift forces acting on each box. In this piece of research, only the bare-deck configuration is studied. Non-structural elements such as traffic barriers and of course aerodynamic appendages such as guide vanes, baffles or wind shields, may change significantly the aerodynamic response of the deck cross section (Ricciardelli et al., 2001; Larsen et al., 2008; Ozkan et al., 2011).

From the engineering design perspective, the problem to address is to conduct a parametric design study, 
in terms of gap distance, which would be of utmost importance at the early design stage in long-span bridge industrial applications. Consequently, a number of gap ratios must be considered and different angles of attack should be studied for each gap. In this work, 14 different gaps are considered, and for each one, 11 different angles of attack are studied, which results in 154 different numerical simulations that must be completed. This means that a relatively high number of CFD simulations must be carried out, hence adopting a relatively inexpensive and robust numerical approach is necessary, in order to deal with a computational burden compatible with the requirements of the initial design stage in industrial applications. Completing an equivalent wind tunnel campaign would have demanded extensive resources in terms of funding and time.

Considering the requirements mentioned in the above paragraph, in the numerical simulations that will be reported next, a 2D approach has been adopted since it is intended to avoid the high computational demands associated with 3D meshes combined with scale-resolving turbulence models that require a large number of cells. Besides, a two-equation turbulence model based on the Boussinesq approximation has been chosen. This turbulence model is Menter's $k-\omega$ SST model (F. Menter et al., 2001), that can be considered nowadays as the standard choice in industrial applications, since it has proven to be accurate in a wide range of problems, and it presents lower computational demands than more sophisticated turbulence models like the ones that specifically model transition or the ones based on the computation of the Reynolds stresses.

Any modelling strategy, numerical or experimental, is affected by some limitations, since it is an engineering approach aiming to replicate the fundamentals of a certain physical phenomenon. Hence, the modelling strategy that has been briefly described in the above paragraphs has certain limitations that must be commented upon, with the aim of properly assessing the results that are going to be reported in the next sections of this article. ( $i$ ) The 2D approach adopted for simulating this problem lacks the ability of considering the 3D phenomena that take place. In fact, the three-dimensionality in the flow has two sources: the 3D geometry of the deck due to the presence of the transverse beams linking the box girders, and the intrinsic 3D behaviour in turbulent flow. The impact of transverse beams in the general aerodynamic response of twin box decks is limited. For instance, the transverse beams have been disregarded in experimental studies such as in Laima et al. (2015) and in the numerical simulations reported in de Miranda et al. (2015). The experimental work by Chen and co-workers (Chen et al., 2014) has shown that transverse beams are responsible for lower fluctuating pressures on the downwind box and the surface facing the gap in the upwind box. The adopted 2D approach is equivalent to impose perfect correlation along the spanwise direction in an equivalent 3D simulation. (ii) The $k-\omega$ SST turbulence adopted herein, in combination with the 2D URANS approach, typically produces single mode large scale unsteady structures without resolving any of the details of turbulence (F. R. Menter, 2009). This gives raise to strong vortices that persist for long time immersed into the global flow. Consequently, in some of the simulations that will be reported, strong large scale vortices impinge upon the downwind box and produce strong pressure fluctuations on the windward surfaces, resulting in fluctuating pressure coefficients higher than the experimental values. In this respect, in de Miranda et al. (2015) it is apparent for the gap widths showing vortex shedding how URANS simulations provide high values for the fluctuating pressures, well above the ones obtained from wind tunnel tests or LES simulations. (iii) Furthermore, according to several authors (Wilcox, 2006; Collie et al., 2008), for transitional boundary layer flows, standard two-equation turbulence models would anticipate transition at a Reynolds number at least one order of magnitude too small. Besides, according to (Langtry et al., 2009), the $k-\omega$ SST turbulence model does not include the capability to properly simulate separation-induced transition when transition develops within the separated shear layer. This would justify the slightly lower size of the separation bubble at the region near the leading edge of the upwind boxes, as well as the lower suctions on the upper sides of both boxes at high positive angles of attack, obtained from the 2D URANS simulations (see $\alpha=+10^{\circ}$ for the Gap K arrangement) as it will be described in the pertinent sections.

In spite of the above mentioned limitations, the ability of the proposed 2D URANS approach to produce valuable information in the frame of parametric design studies at the early stages of industrial projects of long span bridges deserves a detailed assessment. The success in its application would provide engineers with the entire picture concerning the expected aerodynamic behaviour of a twin-box deck bridge as a function of the gap distance at a moderate expense. The above statement relies on the ability of the reported CFD simulations to match the available wind tunnel results. Hence, extensive validation against wind tunnel results is conducted in this work as a tool to show the level of confidence that the designer might give to the numerical results. In fact, the acceptance of CFD-based research by the scientific community relies on the validation of the computational 
results with the experimental data.

\section{Formulation}

The flow around deck cross-sections is modelled by the Navier-Stokes equations, for which time averaging in conservative form yields the Unsteady Reynolds-Averaged Navier-Stokes (URANS) equations (Wilcox, 2006):

$$
\begin{aligned}
& \frac{\partial U_{i}}{\partial x_{i}}=0 \\
& \rho \frac{\partial U_{i}}{\partial t}+\rho U_{j} \frac{\partial U_{i}}{\partial x_{j}}=-\frac{\partial P}{\partial x_{i}}+\frac{\partial}{\partial x_{j}}\left(2 \mu S_{i j}-\rho \overline{u_{i}^{\prime} u_{j}^{\prime}}\right)
\end{aligned}
$$

Where $U_{i}$ is the mean velocity vector, $x_{i}$ is the position vector, $\rho$ is the fluid density, $t$ is the time, $P$ is the mean pressure, $\mu$ is the fluid viscosity, $S_{i j}$ is the mean strain-rate tensor and $u_{i}^{\prime}$ is the fluctuating velocity.

The term $-\overline{u_{i}^{\prime} u_{j}^{\prime}}$ is the so-called specific Reynolds stress tensor $\left(\tau_{i j}\right)$, calculated by means of the Boussinesq assumption as:

$$
\tau_{i j}=2 v_{T} S_{i j}-\frac{2}{3} k \delta_{i j}
$$

Where $v_{T}$ is the kinematic eddy viscosity and $k$ is the kinetic energy per unit mass of the turbulent fluctuation.

Depending on the equations added for the closure of the system, the different URANS models are obtained. In this case, the model used is the $k-\omega \mathrm{SST}$, for incompressible flow, implemented in the open source software OpenFOAM, whose formulation was proposed by F. Menter et al. (2001).

In this work the focus is put on the integral parameters, which are the force coefficients $\left(\right.$ drag $\left(C_{d}\right)$, lift $\left(C_{l}\right)$ and moment $\left.\left(C_{m}\right)\right)$ and the Strouhal number $(S t)$. The force coefficients are calculated time-averaging the results of the integral along the deck surfaces of the pressure distributions and the viscous forces. The Strouhal number is obtained from the frequency content analysis of the $C_{l}$ time history. The force coefficients are calculated as indicated in the equation (4) where $F_{D}, F_{L}$ and $M$ are the time averaged drag and lift forces and moment per unit length. Positive lift and moment are in the upward direction and the clock-wise rotation respectively; finally, positive drag is taken in the along wind direction, which is coincident with the positive $\mathrm{x}$-axis in figure 1.

$$
C_{d}=\frac{F_{D}}{\frac{1}{2} \rho U^{2} C} ; \quad C_{l}=\frac{F_{L}}{\frac{1}{2} \rho U^{2} C} ; \quad C_{m}=\frac{M}{\frac{1}{2} \rho U^{2} C^{2}} ; \quad S t=\frac{f D}{U}
$$

Figure 1: Sign convention.

The mean pressure coefficient $\left(\overline{C_{p}}\right)$ and its standard deviation $\left(\widetilde{C_{p}}\right)$ are calculated as shown by equations $(5)$ and (6). 


$$
\begin{aligned}
& \overline{C_{p}}=\frac{\bar{p}}{\frac{1}{2} \rho U_{\infty}^{2}} \\
& \widetilde{C_{p}}=\frac{\sigma_{p}}{\frac{1}{2} \rho U_{\infty}^{2}}
\end{aligned}
$$

In the latter equations, $\bar{p}$ is the time averaging of the pressure, $\rho$ is the air density, $U_{\infty}$ is the free stream speed and $\sigma_{p}$ is the standard deviation of the pressure. The $C_{p}$ distributions help to understand the resultant mean force coefficients, the fundamental flow features and, for this type of deck cross-sections in conjunction with additional inputs such as flow fields, provide some insights for identifying separation and reattachment regions. Meanwhile the $\widetilde{C_{p}}$ distribution gives an idea of flow separation and reattachment zones, as well as effects of body-induced turbulence (Kwok et al., 2012). 


\section{Modelling and computational approach}

To perform the flow analysis, an overall computational fluid domain of size $(40 C+B) \times 30 C$ was established, where $C$ is the width of one box and $B$ is the overall width of the bridge deck, including the two box girders and the gap $(G)$ between them. $D$ is the deck depth, as can be seen in figure 1 . The size of the computational fluid domain is similar to the one reported in Bruno et al. (2010) for a ratio 5:1 rectangular cylinder. This computational fluid domain has been subdivided into 3 different zones, excluding the boundary layer regions, as shown in figure 2, where the fundamental dimensions are also provided. The zone that is closest to the boxes is the so called buffer zone $(\boldsymbol{L})$, where special care was taken to obtain a high density mesh in the gap between decks. In this region very important phenomena take place, such as: shear layer separation and vortex detachment and impingement. In the same manner, a second region is defined, which is a strip downwind of the buffer zone, which is named as wake zone $(\boldsymbol{M})$. In this region, special care must also be taken in the spatial discretisation, although the cell sizes can be increased as the distance from the deck increases. The wake zone is where the vortices eventually shed from the deck will evolve and progressively dissipate as they are drifted away. Finally, further away from the deck, the zone $(\boldsymbol{N})$ is defined, where coarser cells can be placed. This zone defines the overall size of the fluid domain, which must be large enough in order to avoid the influence of the flow domain boundaries in the flow behaviour in the vicinity of the deck. Pictures of the mesh, including details of the different zones, are presented in figure 3. As can be seen, a non-structured mesh with triangular cells was adopted to discretise the flow domain, except for the boundary layer region, where a quadrangular, structured mesh was used. The height of the boundary layer first element has been chosen in order to get a non-dimensional first layer height target value of $y^{+}=2\left(y^{+}=\left(u_{\tau} y_{1}\right) / \nu ; y_{1}\right.$ is the height of the first element of the boundary layer and $u_{\tau}$ is the friction velocity).

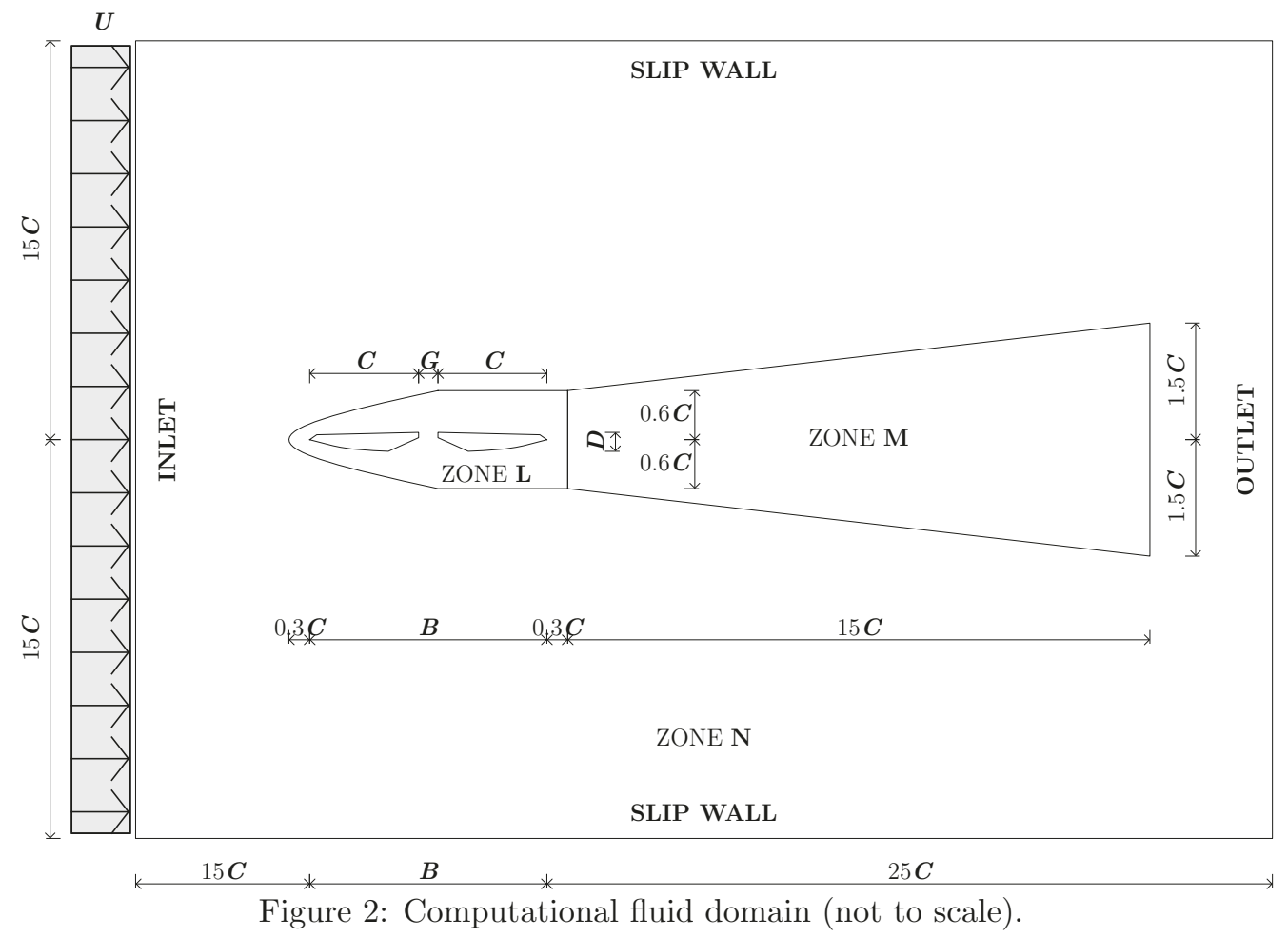




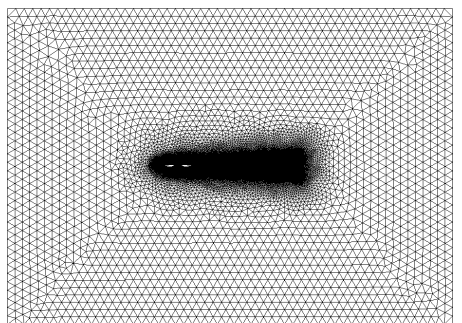

(a) Overall domain.

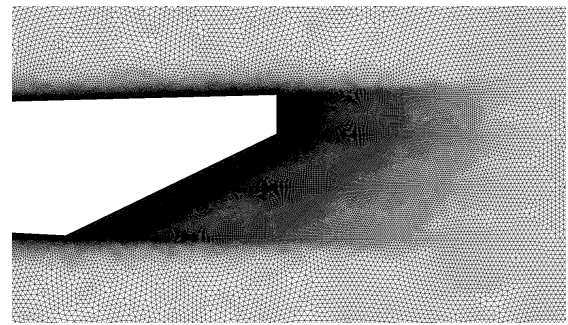

(d) Gap detail 01 .

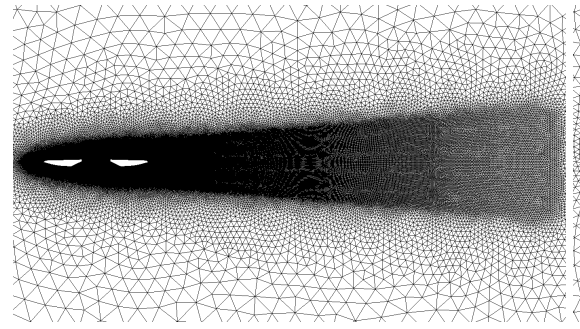

(b) Wake buffer \& wake.

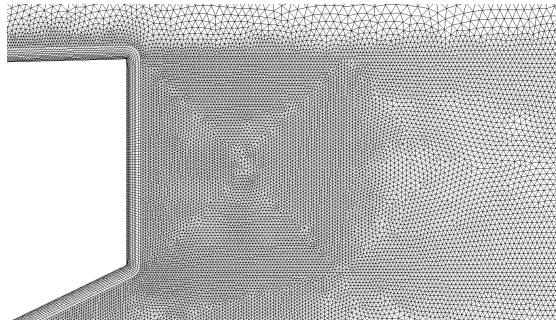

(e) Gap detail 02.

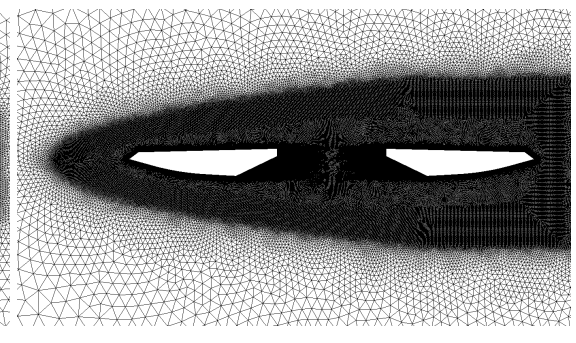

(c) Wake buffer.

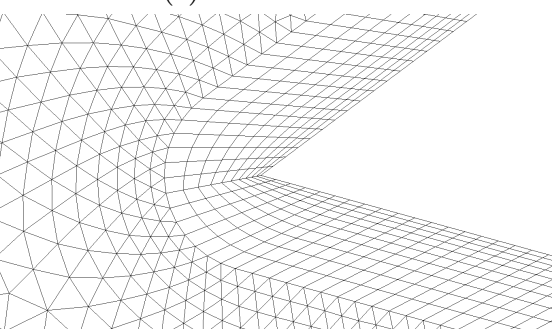

(f) Boundary layer.

Figure 3: Mesh details.

At the inlet (see figure 2), the turbulent intensity of the incoming flow was set to $1 \%$, and the turbulent length scale to $0.1 C$, which are of the same order of magnitude as in Nieto et al. (2015). Dirichlet conditions have been imposed at the inlet for the velocity field $U$, the turbulent specific dissipation $\omega$ and the turbulent kinetic energy $k$. On the other hand, Neuman conditions were considered for the pressure $p$. In the case of the outlet boundary, Neuman conditions were imposed for the velocity, the specific dissipation rate and turbulent kinetic energy fields, meanwhile a Dirichlet condition was set for the pressure field. In the case of the upper and lower boundaries a slip wall boundary condition was selected. Finally, the walls of the boxes are modelled by means of a no-slip boundary condition, therefore, null velocity has been imposed on this boundary.

In some cases, numerical schemes showing first or first-second order behaviour have been favoured aiming to improve the robustness of the simulations that must tackle very different flow conditions depending on the specific gap and angle of attack of each simulation. The open-source finite volume software OpenFoam was used to numerically evaluate the fluid flow around the bridge section. The diffusive terms are computed using a second-order differential scheme, while the convective terms use the linear upwind differential scheme. The advancement in time is accomplished by a first order implicit scheme and the pressure-velocity coupling is resolved by the PIMPLE algorithm. 


\section{Verification and preliminary validation}

In CFD simulations, the finite volume mesh discretisation of the computational domain plays a key role in the accuracy of the results (Mannini et al., 2010). Therefore, a mesh density sensitivity study was carried out. This process tries to identify a mesh in which successive spatial refinements do not produce significant changes in the numerical results. Three meshes, with different densities, have been considered and their properties can be found in table 1 . The differences between meshes in the verification study mainly relay on the successive refinement of the different zones, keeping the same discretisation for the boundary layer, except for the fine mesh, where the length of the elements along the wall in the first layer attached to the twin boxes is half the length adopted in the other meshes. All the simulations in this verification study were carried out at $R e=U_{\infty} D / \nu=4.48 \cdot 10^{4}$, corresponding to a wind speed of $15 \mathrm{~m} / \mathrm{s}$, for a zero degree angle of attack $\left(\alpha=0^{\circ}\right)$. The high number of elements of these meshes is related to the complexity of the geometry studied, in shape and in number of boxes. Furthermore, the downwind box is fully immersed in the wake of the upwind box, which requires a high density mesh in the gap and the vicinity of the downwind box in order to accurately simulate the involved phenomena.

\begin{tabular}{ccccccccc}
\hline Mesh & $y_{1} / C$ & $x_{1} / y_{1}$ & $r$ & $l_{b l}$ & $y_{b l} / C$ & Total cells & Quadrilateral cells & Triangular cells \\
\hline coarse & $1.6432 \cdot 10^{-4}$ & 4 & 1.167 & 10 & $3.5035 \cdot 10^{-3}$ & 413118 & 66820 & 346298 \\
medium & $1.6432 \cdot 10^{-4}$ & 4 & 1.167 & 10 & $3.5035 \cdot 10^{-3}$ & 664032 & 66820 & 597212 \\
fine & $1.6432 \cdot 10^{-4}$ & 2 & 1.167 & 10 & $2.3808 \cdot 10^{-3}$ & 1359262 & 133260 & 1226002 \\
\hline
\end{tabular}

Table 1: Mesh properties.(The parameter $y_{1}$ is the total height of the first element of the boundary layer (bl), $x_{1}$ is the length of first element in the bl, $r$ is the growth ratio of the elements in the bl, $l_{b l}$ is the number of layers forming the bl mesh and $y_{b l}$ is the total height of the bl mesh.)

The values of the $y^{+}$obtained for the left and right boxes are similar to the ones reported in Sarkic et al. (2012) and Nieto et al. (2015), and compatible with the low Reynolds wall modelling approach adopted for this study. In all the cases the percentage of elements with $y^{+}>4$ is lower than $1.2 \%$ and the average value is lower than 2. The highest values are mainly located in the vicinity of the deck corners. A summary of these $y^{+}$values can be found in tables 2 and 3 .

\begin{tabular}{ccccccc}
\hline & \multicolumn{5}{c}{$y^{+}$left deck } \\
Mesh & $y_{\max }^{+}$ & $y_{\text {mean }}^{+}$ & $\% y^{+}>2$ & $\% y^{+}>4$ & $\% y^{+}>6$ & $\% y^{+}>8$ \\
\hline coarse & 7.4359 & 1.9703 & 47.71 & 0.73 & 0.11 & 0.00 \\
medium & 7.5810 & 1.9875 & 43.46 & 0.85 & 0.13 & 0.01 \\
fine & 8.1701 & 1.9822 & 48.18 & 0.75 & 0.12 & 0.02 \\
\hline
\end{tabular}

Table 2: $y^{+}$values for the upwind deck.

\begin{tabular}{ccccccc}
\hline & \multicolumn{5}{c}{$y^{+}$right deck } \\
Mesh & $y_{\max }^{+}$ & $y_{\text {mean }}^{+}$ & $\% y^{+}>2$ & $\% y^{+}>4$ & $\% y^{+}>6$ & $\% y^{+}>8$ \\
\hline coarse & 7.9241 & 1.4557 & 20.30 & 1.13 & 0.11 & 0.01 \\
medium & 7.9865 & 1.4757 & 20.34 & 1.12 & 0.08 & 0.01 \\
fine & 7.8927 & 1.4920 & 20.66 & 1.06 & 0.09 & 0.01 \\
\hline
\end{tabular}

Table 3: $y^{+}$values for the downwind deck. 
The grid sensitivity study is performed based on the values obtained for the integral parameters, that is, the force coefficients and the Strouhal number $(S t)$. The results of the mesh density sensitivity study are presented in table 4 and in figure 4 . It can be appreciated how the results obtained adopting the three different grids are very similar and, particularly the medium and fine meshes provide nearly identical values for the integral parameters. In figure 5, the mean and standard deviation distributions of the pressure coefficient are reported for the reference case (Gap K). It can be found that the differences in the mean and fluctuating pressure distribution are negligible once the coarse mesh is discarded. Hence, the medium mesh characteristics are retained for the remaining simulations given its ability to provide space discretisation independent results with lower computational cost. It must be noted that the cells count is one order of magnitude larger than in the $2 \mathrm{D}$ URANS simulations reported in de Miranda et al. (2015).

\begin{tabular}{cccccccc}
\hline Mesh & $\overline{C_{d}}$ & $\overline{C_{l}}$ & $\overline{C_{m}}$ & $\widetilde{C}_{d}$ & $\widetilde{C_{l}}$ & $\widetilde{C_{m}}$ & $S t$ \\
\hline coarse & 0.143 & -0.264 & 0.021 & 0.05 & 0.43 & 0.12 & 0.26 \\
medium & 0.146 & -0.232 & 0.028 & 0.06 & 0.52 & 0.11 & 0.28 \\
fine & 0.144 & -0.238 & 0.023 & 0.06 & 0.52 & 0.11 & 0.28 \\
\hline
\end{tabular}

Table 4: Results of the grid-refinement study.

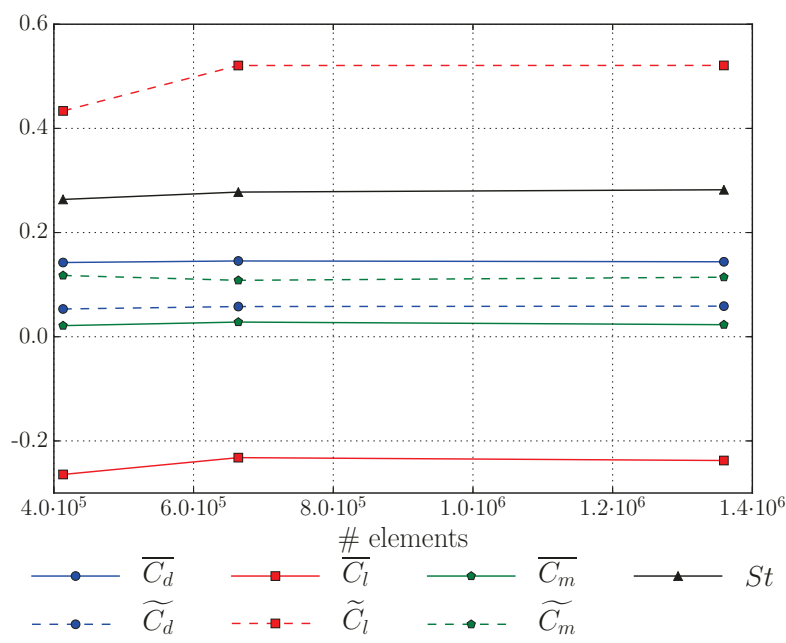

Figure 4: Results of the grid-refinement study. 
All the simulations carried out for the different meshes have been computed imposing a maximum Courant number $(C o)$ of 10 . This number was selected aiming to avoid high computational costs due to the high number of elements in the mesh while satisfying the numerical stability requirements in the simulations. As the time discretisation can also affect the accuracy of the CFD simulations, a sensitivity study imposing different maximum values of $C o(10,5$ and 1$)$ was carried out, adopting the medium mesh spatial discretisation. In this study the results obtained for the different $C o$ numbers considered do not show significant variations, see table 5. Due to the goals of these simulations (analyses of mean force coefficients and $S_{t}$ for a wide range of deck gaps) and the high computational burden associated with a 10 times smaller maximum $C o(C o$ max $=1)$, the medium mesh characteristics and a maximum $C o$ of 10 are retained for the simulation of the other gap to depth ratios considered hereafter.

\begin{tabular}{cccccccc}
\hline Co & $\overline{C_{d}}$ & $\overline{C_{l}}$ & $\overline{C_{m}}$ & $\widetilde{C_{d}}$ & $\widetilde{C_{l}}$ & $\widetilde{C_{m}}$ & $S t$ \\
\hline 10 & 0.146 & -0.232 & 0.028 & 0.06 & 0.52 & 0.11 & 0.28 \\
5 & 0.142 & -0.240 & -0.001 & 0.06 & 0.45 & 0.12 & 0.27 \\
1 & 0.142 & -0.241 & -0.044 & 0.05 & 0.41 & 0.12 & 0.26 \\
\hline
\end{tabular}

Table 5: Time discretisation sensitivity study.

In order to assess the accuracy of the CFD-based results obtained for the reference case, they are compared with the wind tunnel data reported in Kwok et al. (2012) for the equivalent Gap04 arrangement. Since a 2D URANS approach has been adopted in the numerical simulations, 3D geometric details such as the transverse connection beams considered in the tested sectional model cannot be included in the CFD model. Furthermore, the $2 \mathrm{D}$ nature of the simulations prevents the proper modelling of the flow three-dimensionality, as well as the correlation loss in the spanwise direction. In the following the focus will be set on the the mean force coefficients (table 6) and the mean and standard deviation of the pressure coefficients distributions (figure 5).In the CFD simulations, the components of the resultant time-averaged forces and pitching moment per unit of span length are non-dimensionalised using the reference dynamic pressure and a reference length. In the wind tunnel tests reported in Kwok et al. (2012), the mean values of the lift, drag and pitching moment per unit length of the bridge deck are calculated by spatial integration of the mean surface pressure distribution. In order to minimise the random error of the wind tunnel results, force and moment coefficients were averaged over seven pressure-tapped strips located at different positions along the sectional model span.

\begin{tabular}{ccccccccccc}
\hline & Method & $R e$ & $I[\%]$ & $\overline{C_{d}}$ & $\overline{C_{l}}$ & $\overline{C_{m}}$ & $\widetilde{C_{d}}$ & $\widetilde{C_{l}}$ & $\widetilde{C_{m}}$ & $S t$ \\
\hline Present study & $k$ - $\omega$ SST & $4.48 \cdot 10^{4}$ & 1.0 & 0.146 & -0.232 & 0.028 & 0.058 & 0.521 & 0.108 & 0.278 \\
Kwok et al. (2012) & Exp. & $4.48 \cdot 10^{4}$ & - & 0.140 & -0.234 & 0.267 & - & - & - & 0.278 \\
\hline
\end{tabular}

Table 6: Comparison between experimental and numerical values. (All data calculated respect $C$ except for $S t$ calculated respect $D$. I stands for turbulent intensity.)

In both the upwind and downwind boxes, the CFD mean pressure coefficient distribution is very similar to the experimental data from qualitative perspective (see figure 5). In fact, the flow separation and reattachment that takes place along the upper sides of the upwind box is correctly identified, as well as the flow separation at the windward corner of the downwind box. However, looking closer at the values obtained in the upwind box, the CFD simulation provides lower values for the suctions on the upper surface. Furthermore, suctions are overestimated on the windward half of the upper surface of the downwind box, while the suction on the curved side of the downwind box is underestimated. These discrepancies have a limited impact on the mean drag and lift coefficients, which are nearly identical when CFD and experimental values are compared. On the other hand, the aforementioned discrepancies are responsible for the difference obtained in the moment coefficient. The small discrepancies that can be identified in the resultant lift force acting on each individual box are magnified when the moment coefficient is computed introducing the distance from the centre of pressure in each box to the reference axis $\mathrm{O}$ (figure 1).

The CFD simulation provides a resultant lift force acting on the upwind box lower than the wind tunnel 


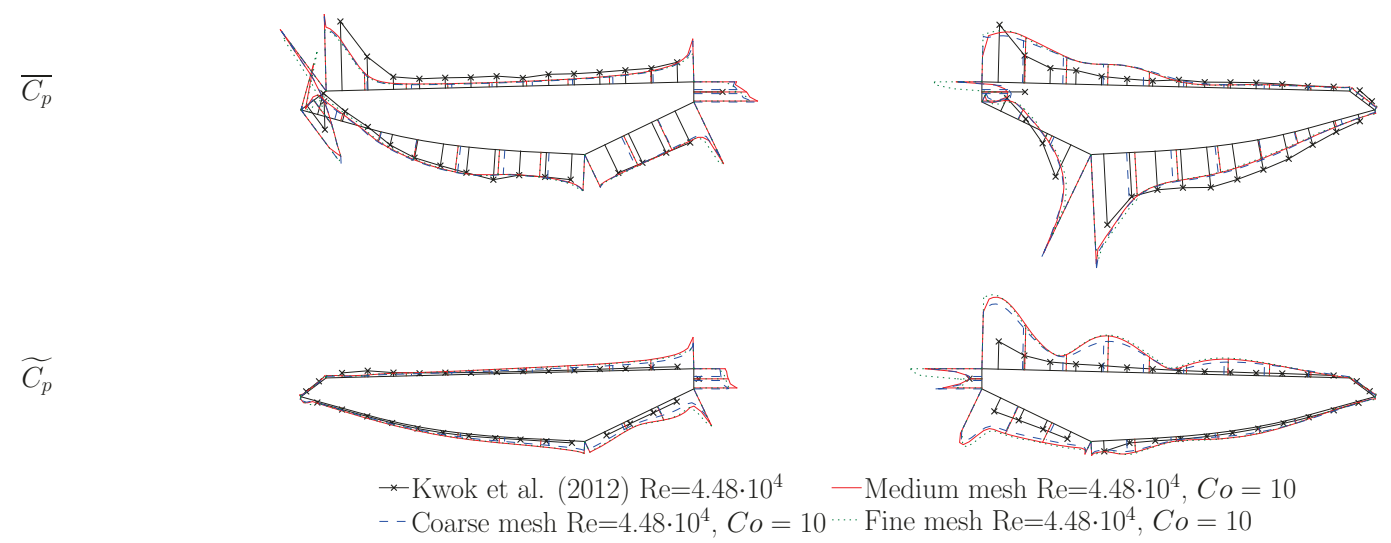

Figure 5: Mean pressure coefficient distribution and its standard deviation distribution for Gap K. (Negative values points outward the section and the height of the section is equal to a value of unity.)

tests. When this lift forces are multiplied by the pertinent lever arm a deficit in the moment coefficient is obtained. In a similar manner, the numerical simulation provides a slightly higher resultant lift force on the downwind box. Consequently the downwind box contribution to the moment coefficient is also lower than in the wind tunnel tests. This explains the lower value obtained in the numerical simulation for the moment coefficient in table 6. Similar discrepancies are reported in the moment coefficient for large gap ratios in the following sections. Similarly a poor agreement in the CFD-based moment coefficients has also been reported in Patruno (2015) for the Gibraltar Bridge case. On the contrary, for small gap to depth ratios, the differences in the moment coefficients are smaller as the shorter lever arm values do not play a multiplicative role when the moment coefficient is computed.

When the distribution of the standard deviation of the pressure coefficient is analysed, it is apparent the higher values provided by the CFD model at the leeward side of the upwind box, and particularly at the windward half of the downwind box. This is due to the generation of strong vortices, typical of two-equation turbulence models, that in this case are shed from the upwind box and impinge upon the downwind box. In de Miranda et al. (2015) similar patterns are obtained for the distributions of the $\widetilde{C_{p}}$ computed from the $2 \mathrm{D}$ URANS simulations.

In terms of Strouhal number the agreement between the CFD value and the wind tunnel result is remarkable. This signals the ability of the proposed 2D URANS approach to simulate the fundamental flow features for static twin box decks.

\section{Results}

The set of simulations presented herein aims to analyse the flow around the bare deck cross-section of the Stonecutters cable-stayed Bridge, depending on the gap to depth ratio, adopting a 2D approach and the $k-\omega$ SST turbulence model. The Stonecutters Bridge is configured as a twin box deck, where two independent box girders with a gap between them, are connected by transversal beams. The width of the individual boxes is $C=19.5 \mathrm{~m}$ and its height is $D=3.609 \mathrm{~m}$, meanwhile the gap distance between the boxes is $G=14.3 \mathrm{~m}, \mathrm{In}$ these CFD simulations, the geometric scale selected to reproduce the section geometry was set to $1 / 80$ and the incoming wind velocity was set to $15 \mathrm{~m} / \mathrm{s}$, aiming to obtain the same Reynolds number as in the experimental tests carried out by Kwok et al. (2012). The arrangement of the actual deck of the Stonecutter Bridge is adopted as the reference case in this work, which corresponds with the Gap04 case in Kwok et al. (2012). This reference case is the one used for verification and preliminary validation, and will be identified as Gap $\mathrm{K}$ in the successive parts of this work (see table 7). 


\subsection{Main flow features, and mean and fluctuating pressure coefficient distributions}

The numerical simulations presented in this paper correspond to fourteen different gap widths at eleven different angles of attack $\left(0^{\circ}, \pm 1^{\circ}, \pm 3^{\circ}, \pm 5^{\circ}, \pm 7^{\circ}, \pm 10^{\circ}\right)$. In table 7 , the different gaps studied with the adopted naming convention and their correspondence with the geometry arrangements studied in Kwok et al. (2012), can be found. The reference case considered for verification and validation, that was previously reported, corresponds to the Gap K arrangement in table 7 . The meshes that have been built for the cases considered herein are based on the characteristics of the medium mesh of the reference case, previously described.

\begin{tabular}{|c|c|c|c|c|c|c|c|c|c|c|c|c|c|c|}
\hline Gap & $\mathrm{A}$ & $\mathrm{B}$ & $\mathrm{C}$ & $\mathrm{D}$ & $\mathrm{E}$ & $\mathrm{F}$ & $\mathrm{G}$ & $\mathrm{H}$ & $\mathrm{I}$ & $\mathrm{J}$ & $\mathrm{K}$ & $\mathrm{L}$ & $\mathrm{M}$ & $\mathrm{N}$ \\
\hline$G / D$ & 0.00 & 0.28 & 0.55 & 0.83 & 1.11 & 1.25 & 1.66 & 2.08 & 2.35 & 2.63 & 3.96 & 5.85 & 7.48 & 9.70 \\
\hline Kwok et al. (2012) & 01 & 02 & - & - & - & - & - & 03 & - & - & 04 & 05 & - & - \\
\hline
\end{tabular}

Table 7: Name convention of the investigated gap ratios and its correspondence with the gap identification codes in Kwok et al. (2012).

In the following, snapshots of the instantaneous vorticity fields, and the distributions of the mean pressure coefficient $\left(\overline{C_{p}}\right)$ and its standard deviation $\left(\widetilde{C_{p}}\right)$, are presented and discussed for four different gap widths, representative of the different flow patterns appreciated, at an angle of attack of $0^{\circ}$. Besides, for the Gap $\mathrm{K}$ configuration, the results of the CFD simulations for the challenging cases of $\pm 10^{\circ}$ angles of attack are reported and compared with the available wind tunnel data. The Reynolds number used in all the numerical simulations is the same as the one used in the experimental studies in Kwok et al. (2012), whose results are presented for comparison and validation, being equal to $R e=4.48 \cdot 10^{4}$.

The main goal of this section is the identification of the fundamental flow features and their changes as the slot between boxes increases. Instantaneous vorticity fields allow to identify vortices and shear layers, along with their evolution in time. Mean and fluctuating pressure coefficients are useful to identify flow separation and reattachment, as well as vortex impingement on the downwind box. The comparison of the aforementioned mean and fluctuating pressure coefficient distributions with the equivalent wind tunnel results helps to judge the feasibility of the numerical simulations and also facilitates the understanding of the causes responsible for the eventual discrepancies between CFD and experimental data.

\subsubsection{Gap A, $\alpha=0^{\circ}$}

Gap A $(G / D=0)$ corresponds with Gap01 in Kwok et al. (2012). The CFD simulations for this case provide a steady response similar to the results in Kwok et al. (2012). In figure 6 the vorticity field is presented, where the steady shear layers are apparent. Figure 7 shows the distributions of the mean and fluctuating pressure coefficients. The agreement between the computed and experimental $\overline{C_{p}}$ is fairly good, being the main flow features described in Kwok et al. (2012) correctly predicted by the CFD simulations. Small fluctuations in the experimental pressure coefficient on the inclined panel facing the gap at the downwind box can not be identified due to the steady response obtained from the 2D CFD simulation.

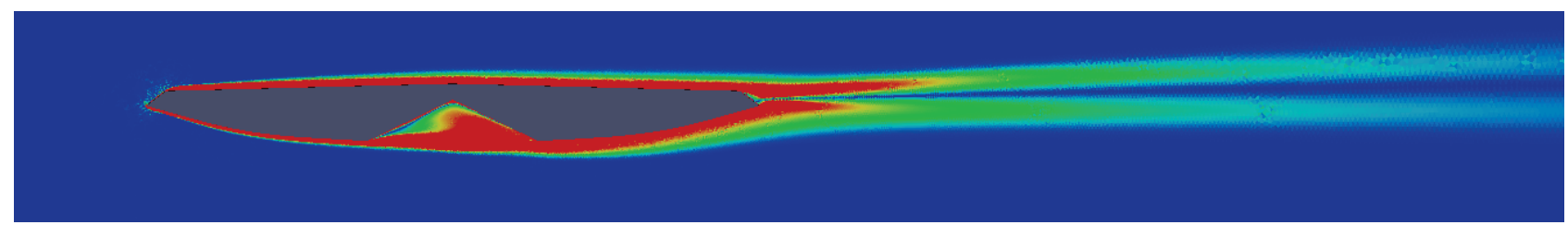

Figure 6: Vorticity magnitude field for Gap A at $0^{\circ}$ angle of attack. 


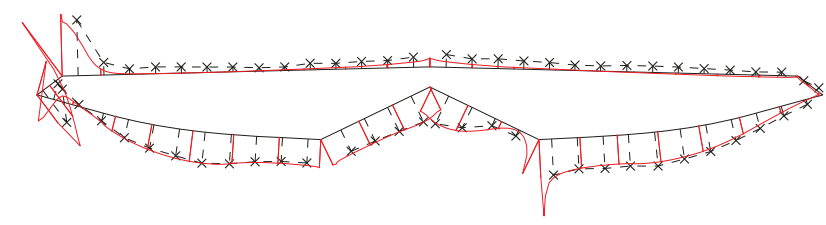

$$
\widetilde{C_{p}}
$$

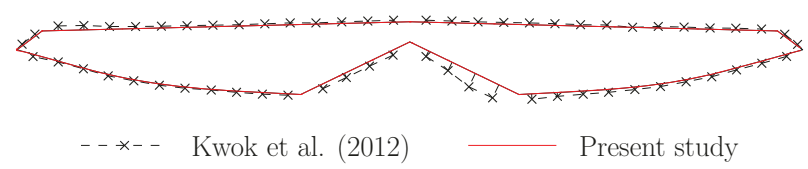

Figure 7: $\overline{C_{p}}$ and $\widetilde{C_{p}}$ distributions for Gap A and Gap01 at $0^{\circ}$ angle of attack. (Negative values points outward the section and the height of the section is equal to a value of unity.)

\subsubsection{Gap B, $\alpha=0^{\circ}$}

Gap B $(G / D=0.28)$ is the same deck arrangement as Gap02 in Kwok et al. (2012). This case corresponds to the two boxes presenting the smallest gap between them. In figure 8,10 snapshots of the instantaneous vorticity fields along one period of the lift coefficient time history are presented. It is possible to identify the formation of a strong vortex, which detaches from the lower leeward corner of the upwind box and impinges upon the inclined windward panel of the downwind box, moving afterwards along the lower side of the downwind box. In the same manner, it can be appreciated the unsteady flow passing through the gap due to the pressure difference between the top and bottom surfaces of the deck. This pressure difference is generated by the accelerated flow moving over the more streamlined bottom surface, according with Bernoulli's Principle as it is described in Kwok et al. (2012). Furthermore, it is evident the weak vortex shedding pattern behind the downwind box, which is consistent with the so-called pattern A behaviour described in Laima et al. (2015) for twin-box decks with low gap to depth ratios.

The comparison between CFD and experimental mean and fluctuating pressure coefficient distributions along the boxes presented in figure 9 , shows a good general agreement, particularly for the $\overline{C_{p}}$. The numerical simulation consistently replicates the main flow features such as the flow separation at the top surface near the leading edge region and the subsequent reattachment, as well as the negative pressures along the bottom surfaces, which have been extensively described in Kwok et al. (2012). The main discrepancies can be found at the surfaces of the boxes facing the gap, where higher values for the standard deviation of the pressure coefficient are obtained in the numerical simulations. This circumstance is due to the perfect spanwise correlation implicit in 2D CFD modelling and the strong vortices typical of two-equation turbulence models, which give place to strong oscillations in pressure when the vortices detach or impinge on a downwind surface. 


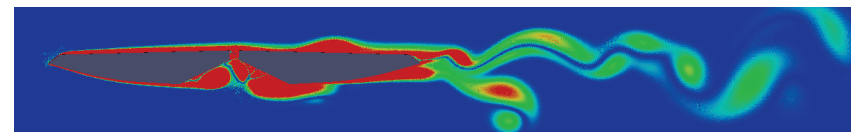

(a) P01

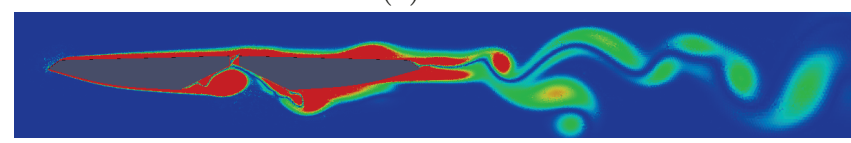

(c) P03

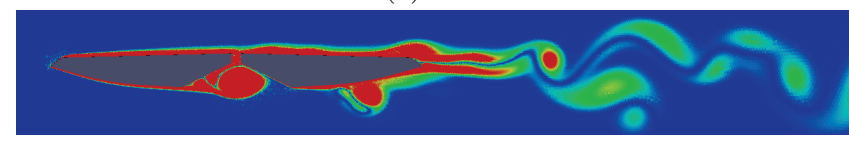

(e) P05

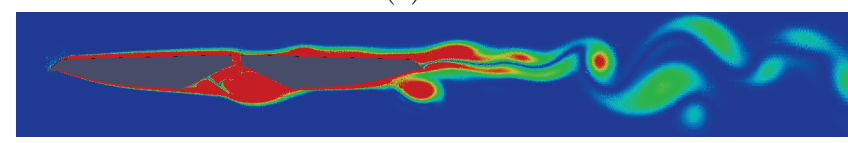

(g) P07

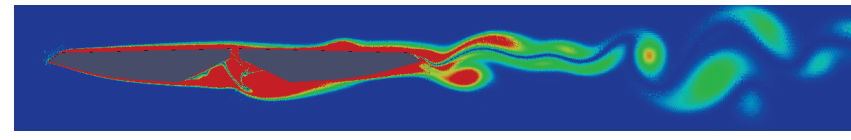

(i) P09

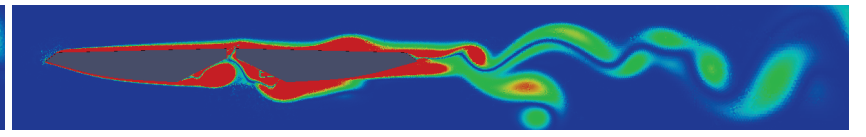

(b) P02

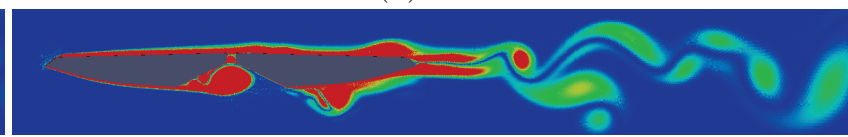

(d) P04

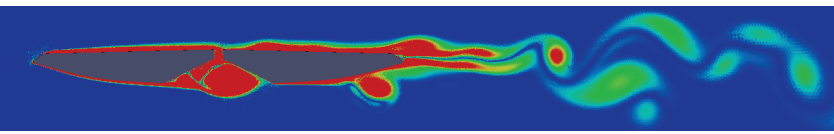

(f) P06

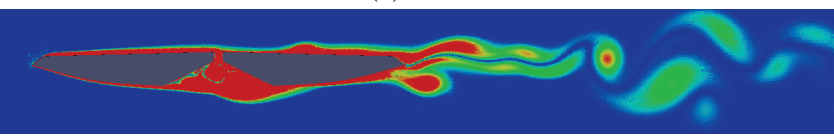

(h) P08

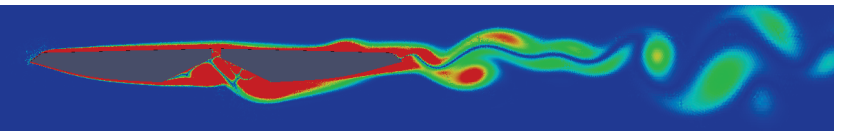

(j) P10
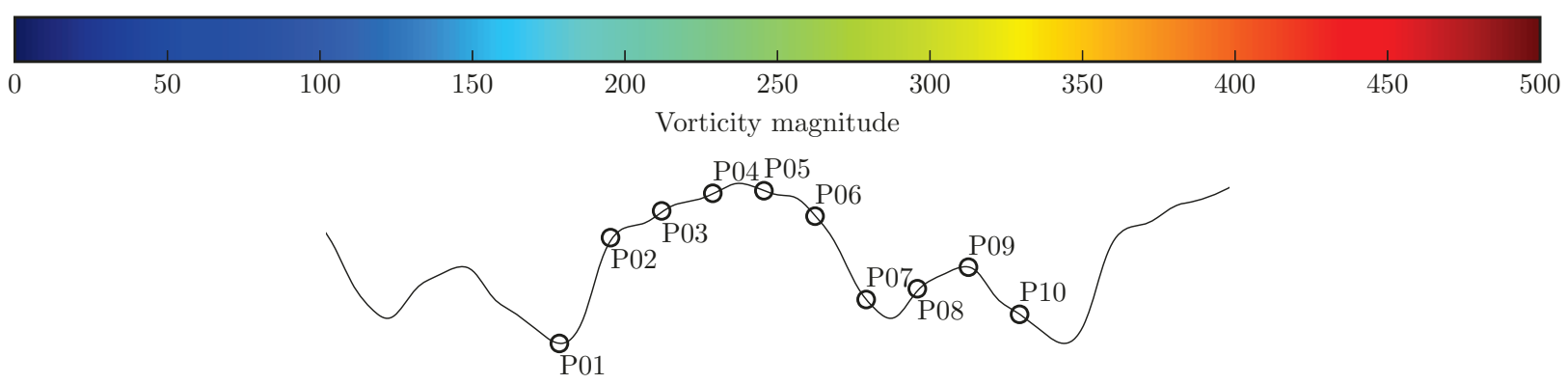

(k) Positions of the vorticity field snapshots in a cycle of the $C_{l}$ time-history.

Figure 8: Vorticity magnitude field snapshots at different positions in a cycle of the $C_{l}$ time-history for the Gap B.

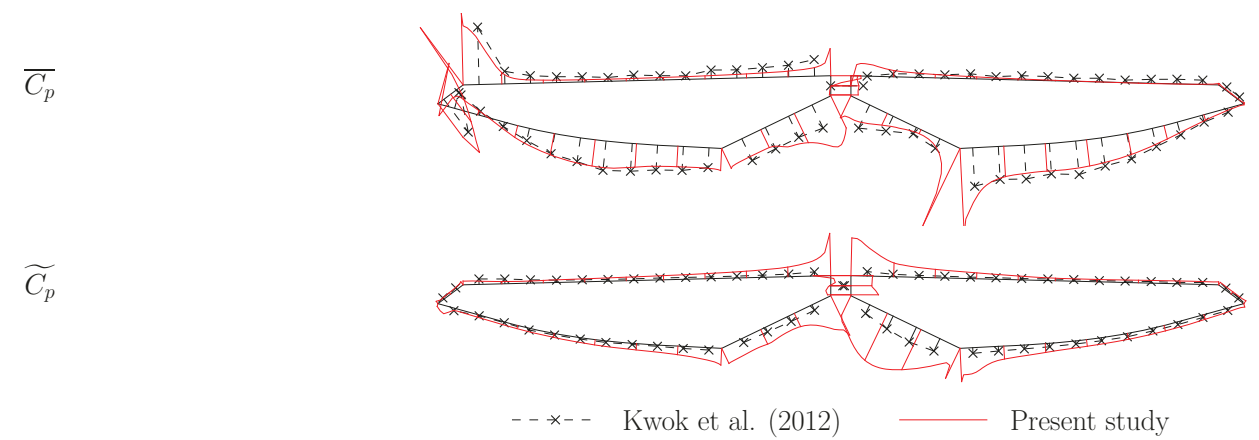

Figure 9: $\overline{C_{p}}$ and $\widetilde{C_{p}}$ distributions for Gap B and Gap02 at $0^{\circ}$ angle of attack. (Negative values points outward the section and the height of the section is equal to a value of unity.) 


\subsubsection{Gap $\mathbf{H}, \alpha=0^{\circ}$}

The next gap to be studied is the Gap $\mathrm{H}(G / D=2.08)$ corresponding with the Gap03 in Kwok et al. (2012).This gap to depth ratio is representative of the aerodynamic behaviour at the transition between short and large gap ratios. In fact, the value $G / D=2.08$ is very close to the value of 2.138 that separates Pattern A and Pattern B vortex-shedding behaviours reported in Laima et al. (2015). Consequently, in the snapshots reported in figure 10 the relatively weak vortex shedding in the wake of the downwind box (pattern A) co-exists with the clear von Karman street in the gap caused by the vortices alternatively shed from the upwind box (pattern B). As the gap width increases, vortices in the gap have a larger distance to develop and the impinging vortices roll over the upper surface of the downwind box. Consequently the mean suction over the top surface is increased, particularly at the windward corner due to the strength of these vortices that further induces flow separation at the top corner facing the gap. The values of $\widetilde{C_{p}}$ over the whole length of the top surface also increase due to the vortices moving along this side of the box. Turning the focus to the comparison between the numerical and experimental distributions of $\overline{C_{p}}$, they show a good agreement. It is argued that the overestimation of the $\widetilde{C_{p}}$ around the windward faces of the downwind box is related to the intrinsic characteristics of the $k-\omega$ SST turbulence model, as previously explained.

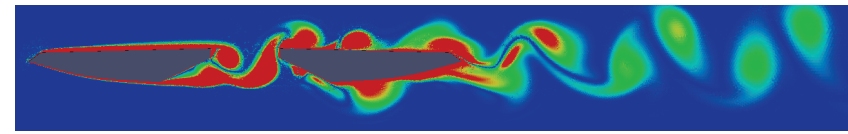

(a) P01

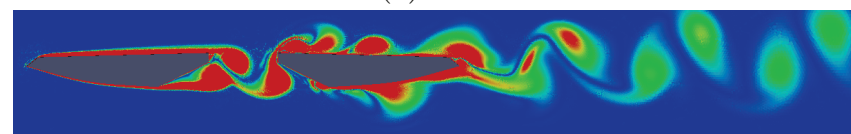

(c) P03

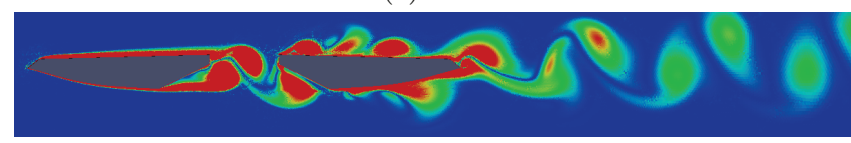

(e) P05

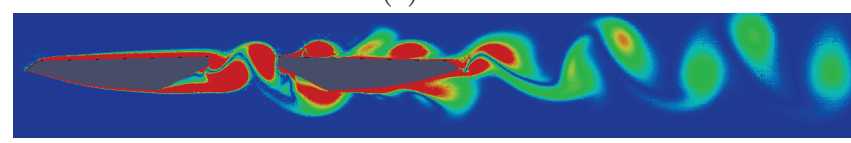

(g) P07

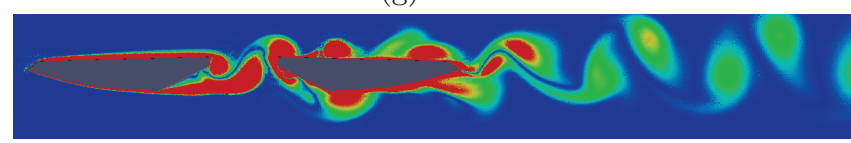

(i) P09

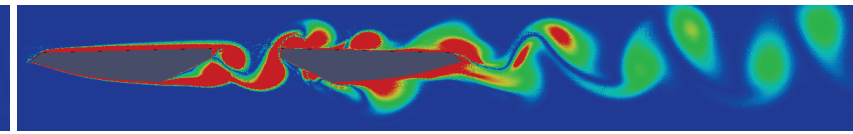

(b) P02

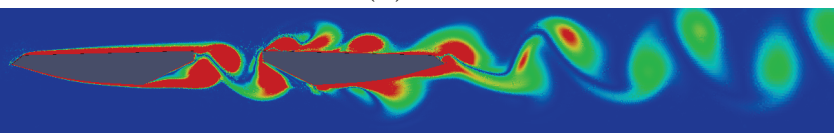

(d) P04

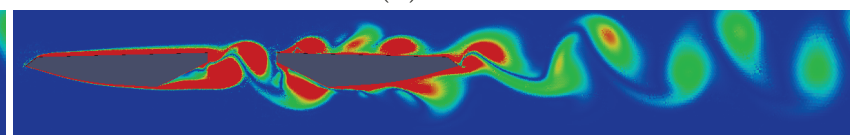

(f) P06

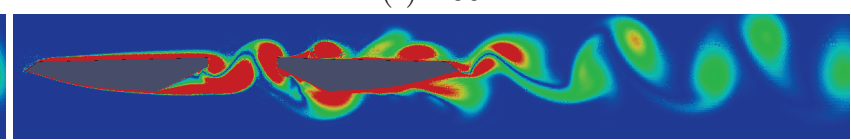

(h) P08

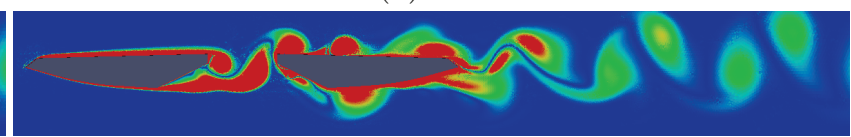

(j) P10
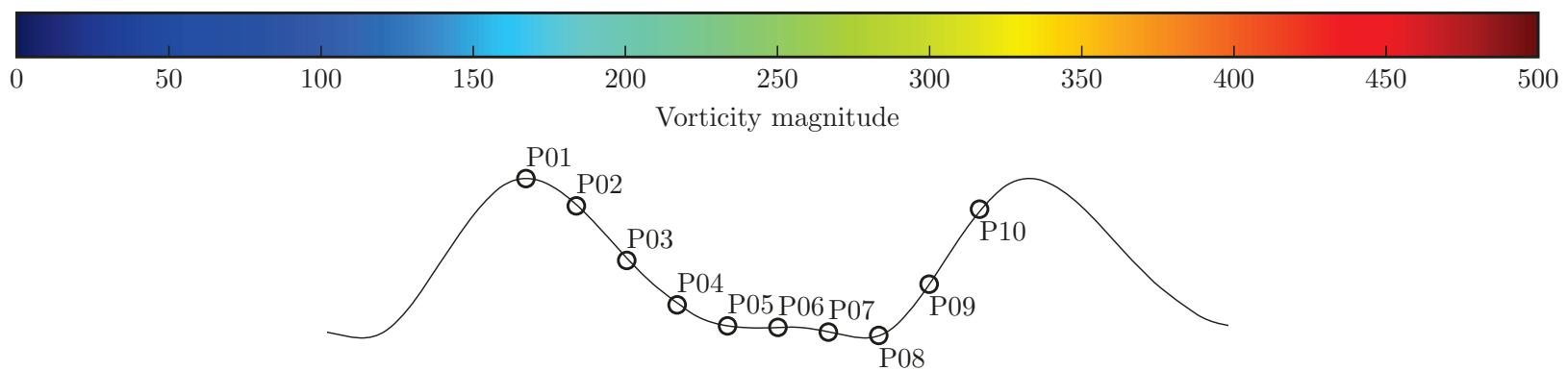

(k) Positions of the vorticity field snapshots in a cycle of the $C_{l}$ time-history.

Figure 10: Vorticity magnitude field snapshots at different positions in a cycle of the $C_{l}$ time-history for the Gap H. 


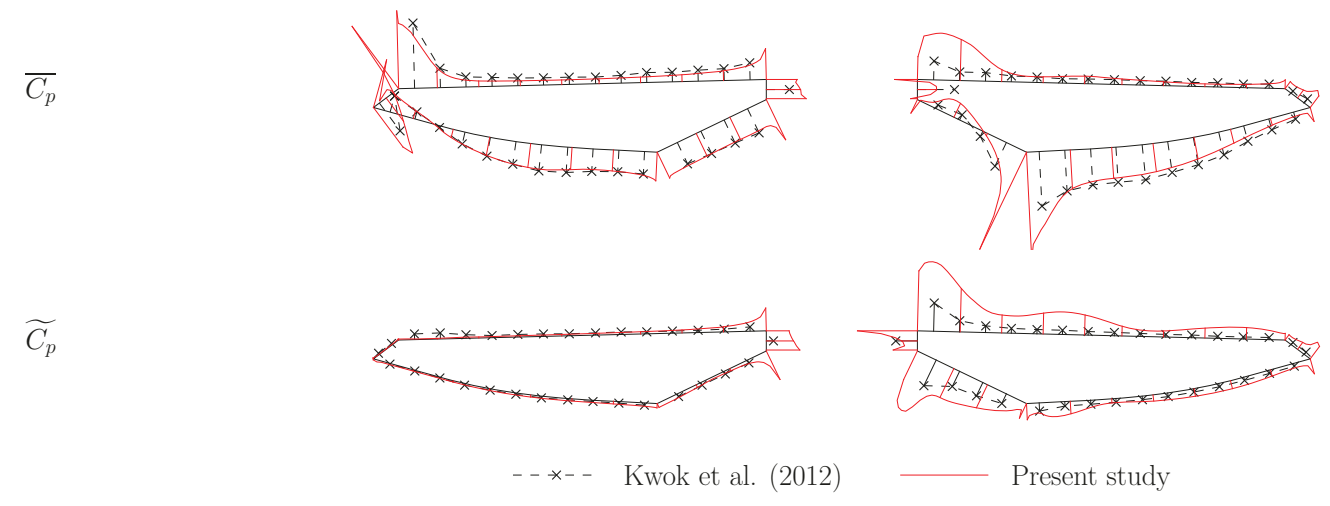

Figure 11: $\overline{C_{p}}$ and $\widetilde{C_{p}}$ distributions for Gap H and Gap03 at $0^{\circ}$ angle of attack. (Negative values points outward the section and the height of the section is equal to a value of unity.) 


\subsubsection{Gap $\mathbf{K}, \alpha=0^{\circ}$}

For this gap to depth ratio $(G / D=3.96)$, which is the one adopted in the verification and preliminary validation studies, the snapshots of instantaneous vorticity are reported in figure 12 for $\alpha=0^{\circ}$. It is apparent how the larger gap allows the complete development of the vortices alternatively shed from the upwind box. This vortices impinge on the downwind box and persist for some time, moving along the top and bottom surfaces of the downwind box. From these pictures no vortex shedding can be identified at the wake of the downwind box, which is fully consistent with the pattern B vortex shedding behaviour described in Laima et al. (2015).

In figure 13 the $\overline{C_{p}}$ and $\widetilde{C_{p}}$ distributions for Gap $\mathrm{K}$ at $0^{\circ}$ angle of attack are presented. The $\overline{C_{p}}$ numerical results are in good agreement with the experimental ones and the differences in the mean $C_{p}$ distributions were already commented upon in section 4. Here, it is noted that the overestimation in the suction on the first half of the upper face of the downwind box may be explained by the large scale, high strength vortices produced by the turbulence model which impinge upon the upper leading edge of the downwind deck. Finally, the overestimation in the $\widetilde{C_{p}}$ distributions is also due to the highly energetic vortices shed from the upwind box.

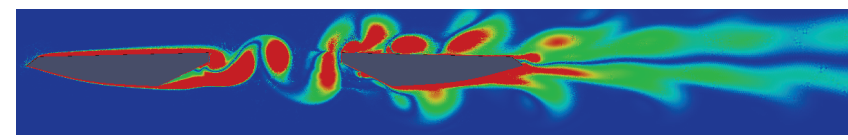

(a) P01

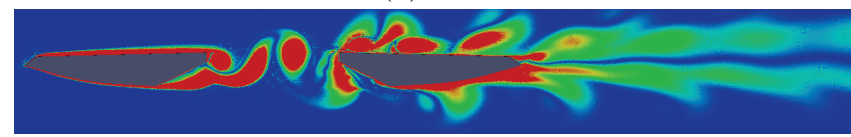

(c) P03

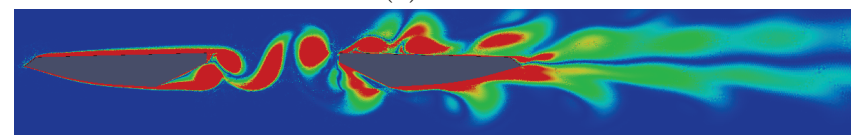

(e) P05

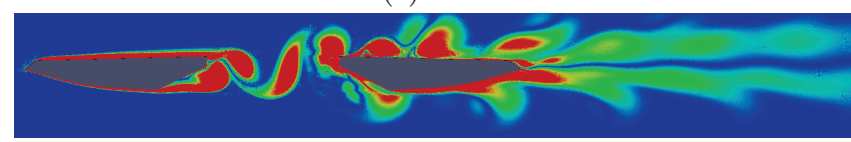

(g) P07

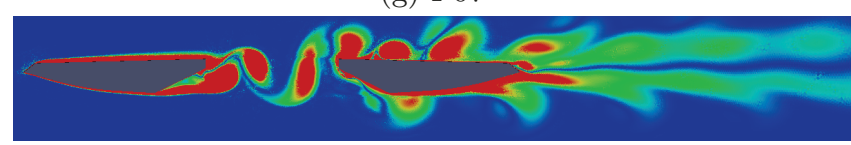

(i) P09

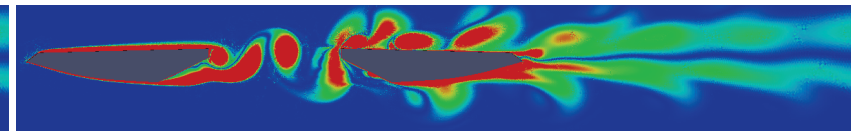

(b) P02

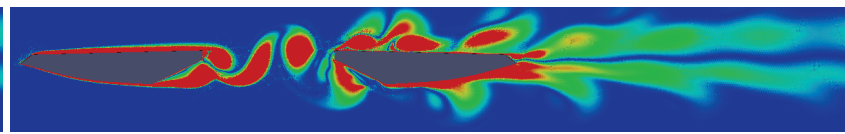

(d) P04

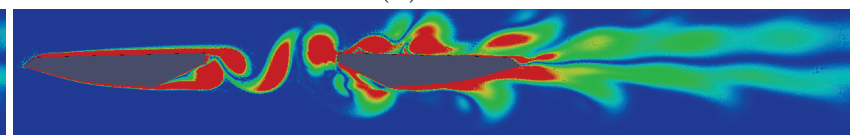

(f) P06

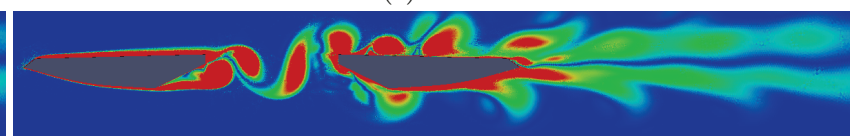

(h) P08

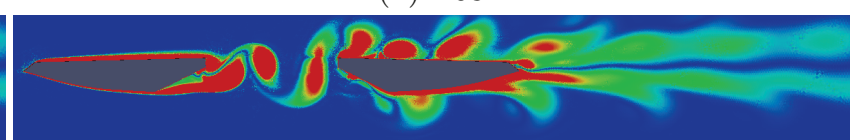

(j) P10
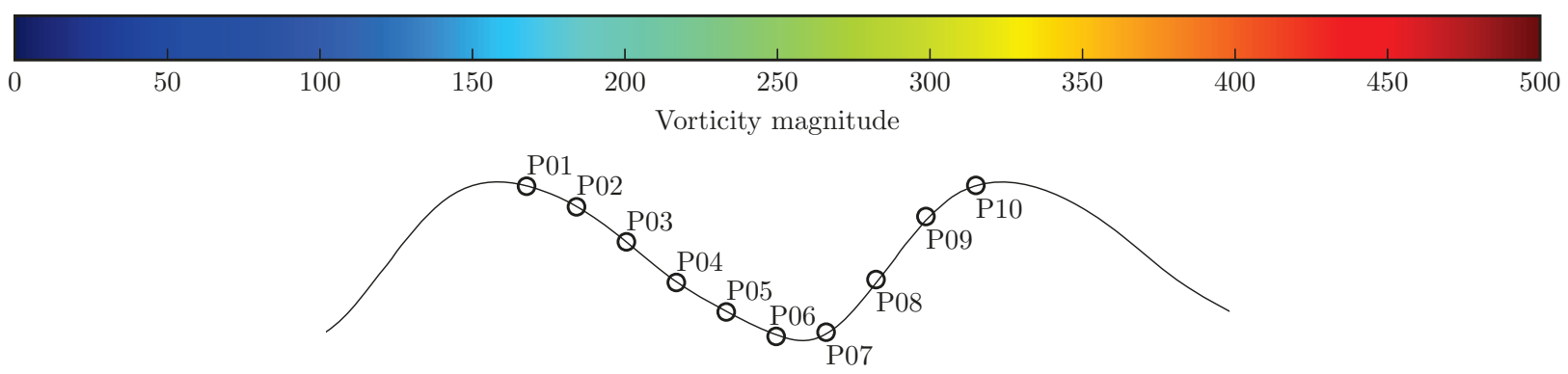

(k) Positions of the vorticity field snapshots in a cycle of the $C_{l}$ time-history.

Figure 12: Vorticity magnitude field snapshots at different positions in a cycle of the $C_{l}$ time-history for the Gap K. 

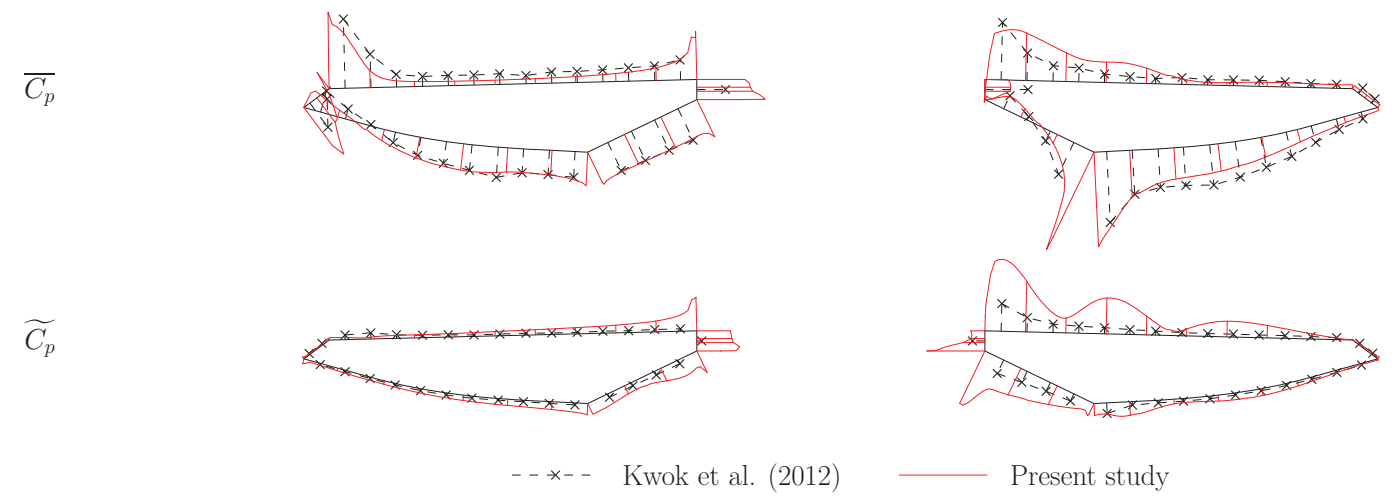

Figure 13: $\overline{C_{p}}$ and $\widetilde{C_{p}}$ distributions for Gap $\mathrm{K}$ and Gap04 at $0^{\circ}$ angle of attack. (Negative values points outward the section and the height of the section is equal to a value of unity.)

\subsubsection{Gap $\mathbf{K}, \alpha=-10^{\circ}$}

In Kwok et al. (2012) the experimental $\overline{C_{p}}$ and $\widetilde{C_{p}}$ distributions at angles of attack $\alpha=-10^{\circ}$ and $\alpha=+10^{\circ}$ have been reported. It is of great interest in this piece of research to compare the CFD results with the wind tunnel ones at high angles of attack. These high angles of attack pose a particularly demanding scenario for CFD simulations since massive flow separation and complex interaction of the upwind box wake with the downwind box are expected.

In figure 14 it can be appreciated the massive flow separation that exists at the upwind box, which gives place to huge vortices that are alternatively shed. In fact, the vortices shed from the upper leeward corner of the upwind box are responsible for the high standard deviation in the pressure along the inclined panel in the downwind box that are obtained from the CFD simulations (figure 15). In figure 14, it can be appreciated how vortices are also alternatively shed from downwind box. In the same manner, pressure fluctuations are smaller over the top surface of the downwind box since the CFD model predicts a partial impingement on the downwind box of the vortices shed from the leeward corner of the upwind box. In the numerical model, the trajectory of that vortex seems to be notably influenced by the other enormous vortex, which is shed from the windward corner of the upwind box, that apparently pushed the former towards the downwind box. Furthermore, the aforementioned vortices seem to interact with the ones detached from the upwind corner of the downwind box since both seem to drift away almost simultaneously, without coalescing.

In figure 15 it can be seen the remarkably good agreement obtained between the experimental and numerical mean pressure coefficient distribution. The upper sides of both boxes must withstand positive mean pressure since at $\alpha=-10^{\circ}$ these plates are facing the flow. Only at the leeward corners moderate negative mean pressures are apparent due to the vortex formation. In the wind tunnel tests, the $\widetilde{C_{p}}$ is not so high over the inclined panel of the downwind box. This might be indicative of a lower size and energy in the vortical structures, but also a different trajectory in the vortices shed from the upper leeward corner of the upwind box. 


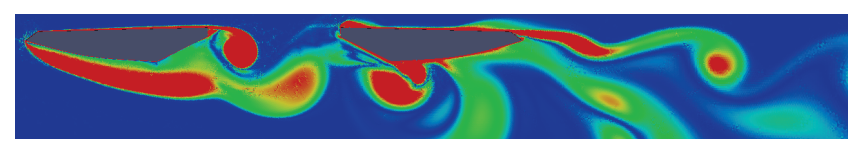

(a) P01

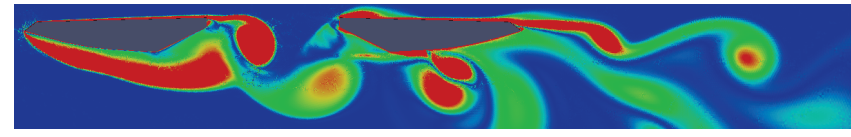

(c) P03

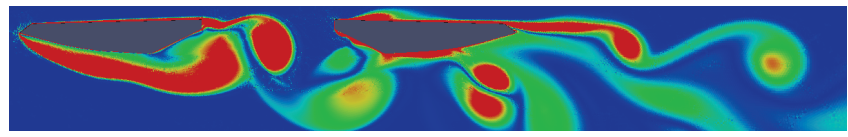

(e) P05

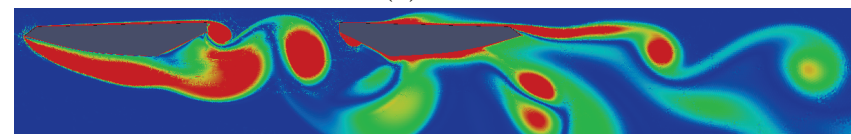

(g) P07

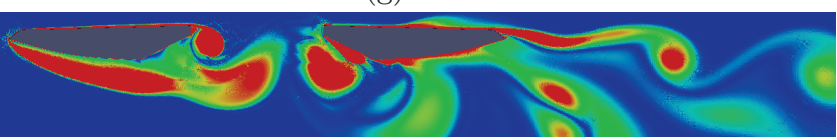

(i) P09

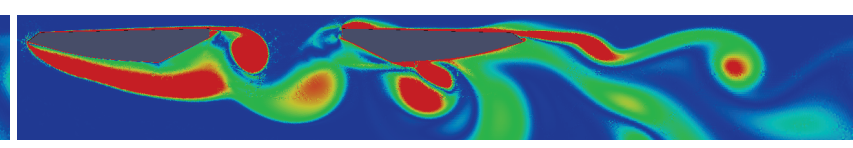

(b) P02

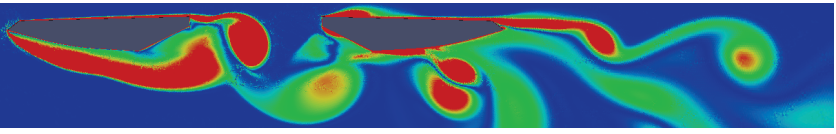

(d) P04

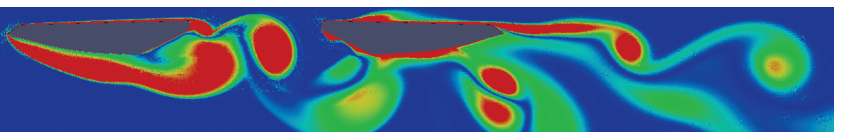

(f) P06

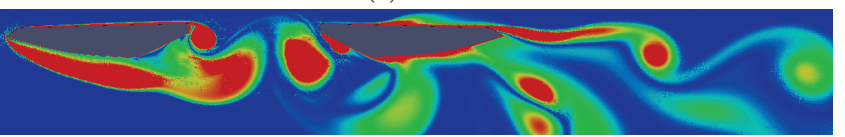

(h) P08

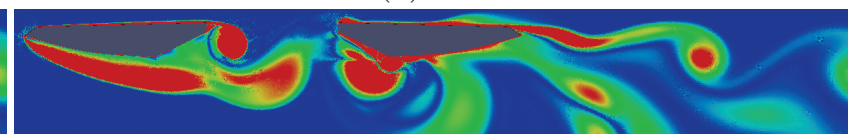

(j) P10
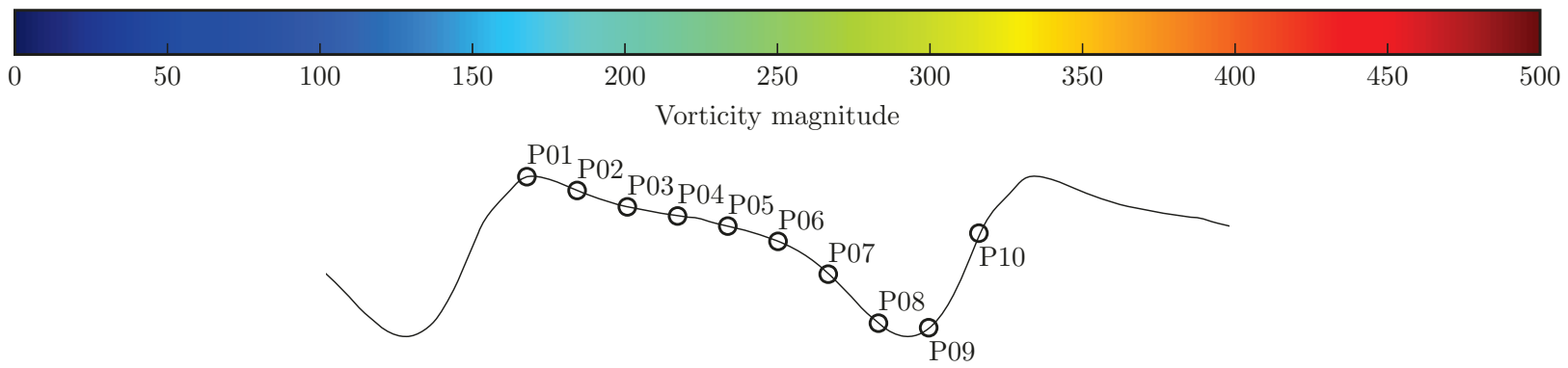

(k) Positions of the vorticity field snapshots in a cycle of the $C_{l}$ time-history.

Figure 14: Vorticity magnitude field snapshots at different positions in a cycle of the $C_{l}$ time-history for the Gap K at $-10^{\circ}$ angle of attack.
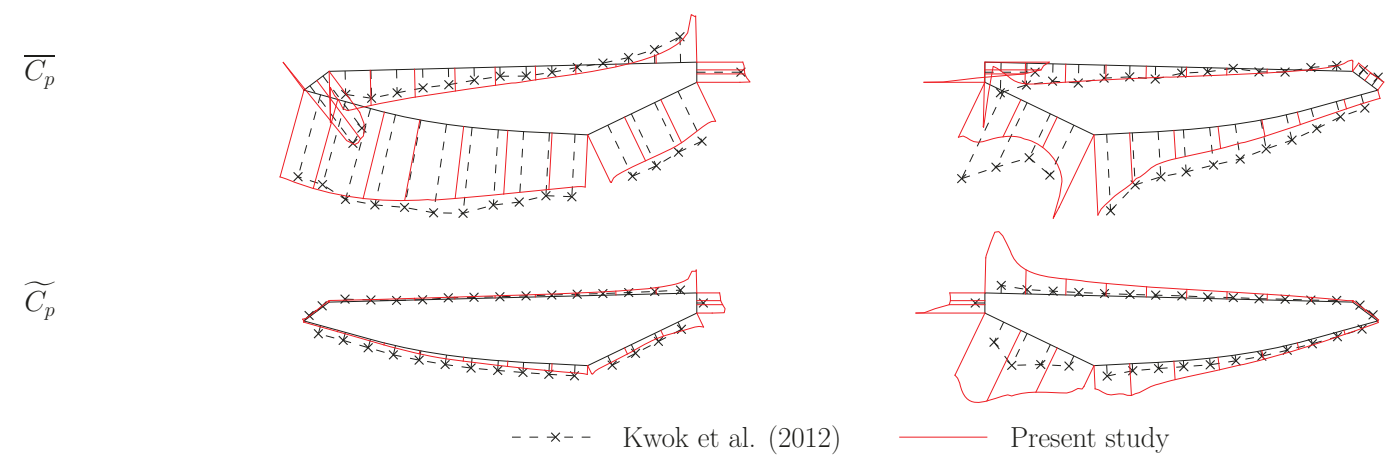

Figure 15: $\overline{C_{p}}$ and $\widetilde{C_{p}}$ distributions for Gap K and Gap04 at $-10^{\circ}$ angle of attack. (Negative values points outward the section and the height of the section is equal to a value of unity.) 


\subsubsection{Gap K, $\alpha=10^{\circ}$}

In figure 16, the snapshots of instantaneous vorticity fields along one period of the lift coefficient time history for the $10^{\circ}$ angle of attack case are provided. As in the latest case, massive flow separation takes place; however, the large shear layers that appear in the upwind box do not roll over giving place to vortices. Furthermore, the shear layer created when the flow separates at the corner of the bottom surface of the upwind box extends up to the downwind box and passes through the gap, interacting with the shear layer created as the flow separates at the windward top corner of the downwind box. In the numerical simulation, vortices are alternatively shed from the downwind box, though the centre of these vortices is far away from the top surface of the downwind box.

When the mean pressure coefficient distribution obtained by means of the 2D URANS simulation is compared with the wind tunnel data (figure 17), it is apparent the inability of the numerical simulation to accurately simulate the strong suctions that take place over the top surfaces of these boxes. The absence of the peak in the pressure suction in the vicinity of the windward corner of the downwind box may be related with the feeding of energy into the shear layer caused by the shear layer detached from the bottom surface of the upwind box that makes the vortex generation to happen farther away from the box top surface. Furthermore, as it has already been mentioned in the introduction, two-equation turbulence models face difficulties for accurately simulate transition in boundary layer flows and separation-induced transition in shear layers. This may explain the long shear layers created at the separation points and the steadiness of the shear layer created at the windward corner of the upwind box, which should have showed some unsteadiness and probably vortex formation according to the pattern in the experimental values of the fluctuating pressure coefficient over the top surface of the upwind box.

This is a particularly challenging case for CFD simulations. The discrepancies are constrained to the values of the suctions on the top surfaces of the deck, while the values of the mean pressure coefficients over the bottom surfaces and the fluctuating pressure coefficients, with the exception mentioned above, show a remarkable agreement with wind tunnel data. Hence, it can be concluded that the 2D URANS simulations reported herein are able to accurately simulate time-averaged quantities for twin-box decks considering different gap distances in a wide range of angles of attack $\left(-10^{\circ}, 5^{\circ}\right)$. Only for high positive angles of attack $\left(7^{\circ}, 10^{\circ}\right)$, the accuracy in the numerical values diminishes, while the results keep on being qualitatively correct. The extent and impact of these differences between numerical and experimental values is made clear when the complete set of force coefficients is reported in section 5.2. It will be demonstrated that the assessment of the aerodynamic performance of the considered deck geometries can be successfully conducted adopting the proposed computational approach. 


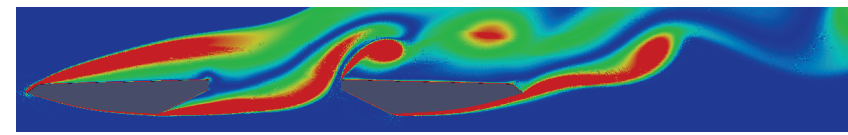

(a) P01

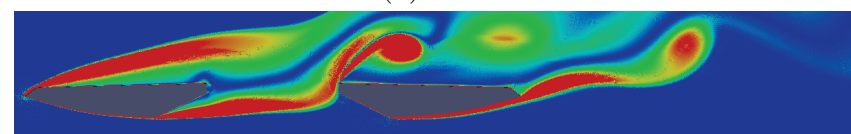

(c) $\mathrm{P} 03$

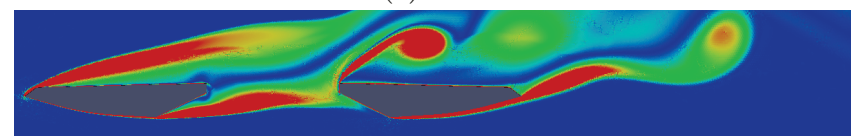

(e) P05

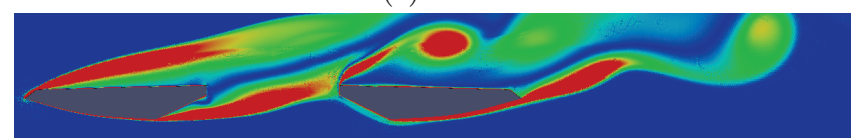

(g) $\mathrm{P} 07$

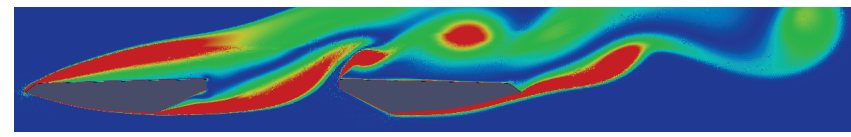

(i) P09

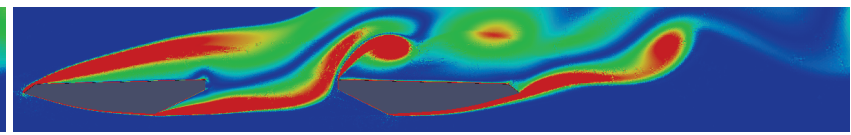

(b) $\mathrm{P} 02$

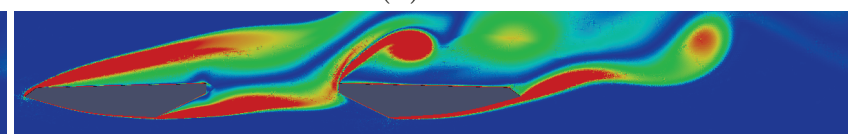

(d) $\mathrm{P} 04$

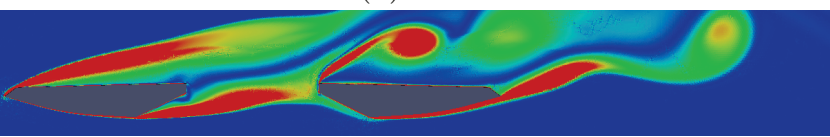

(f) P06

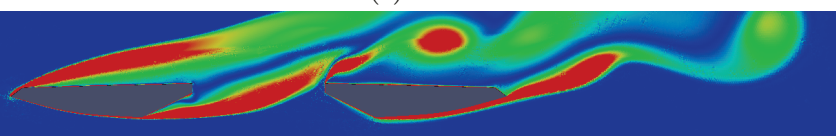

(h) P08

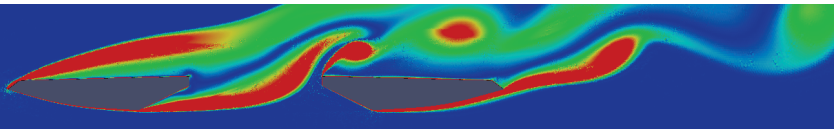

(j) P10
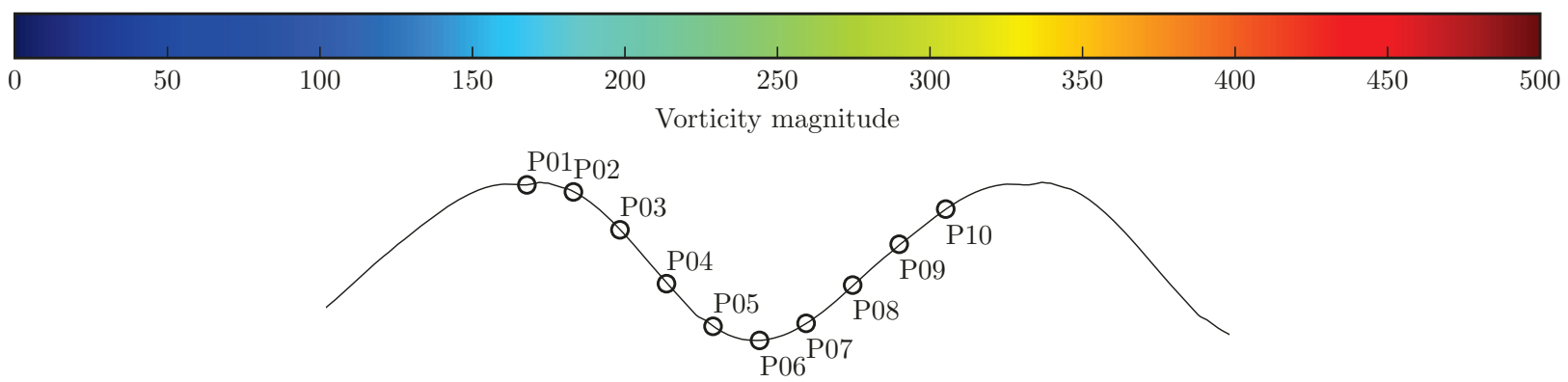

(k) Positions of the vorticity field snapshots in a cycle of the $C_{l}$ time-history.

Figure 16: Vorticity magnitude field snapshots at different positions in a cycle of the $C_{l}$ time-history for the Gap K at $+10^{\circ}$ angle of attack..

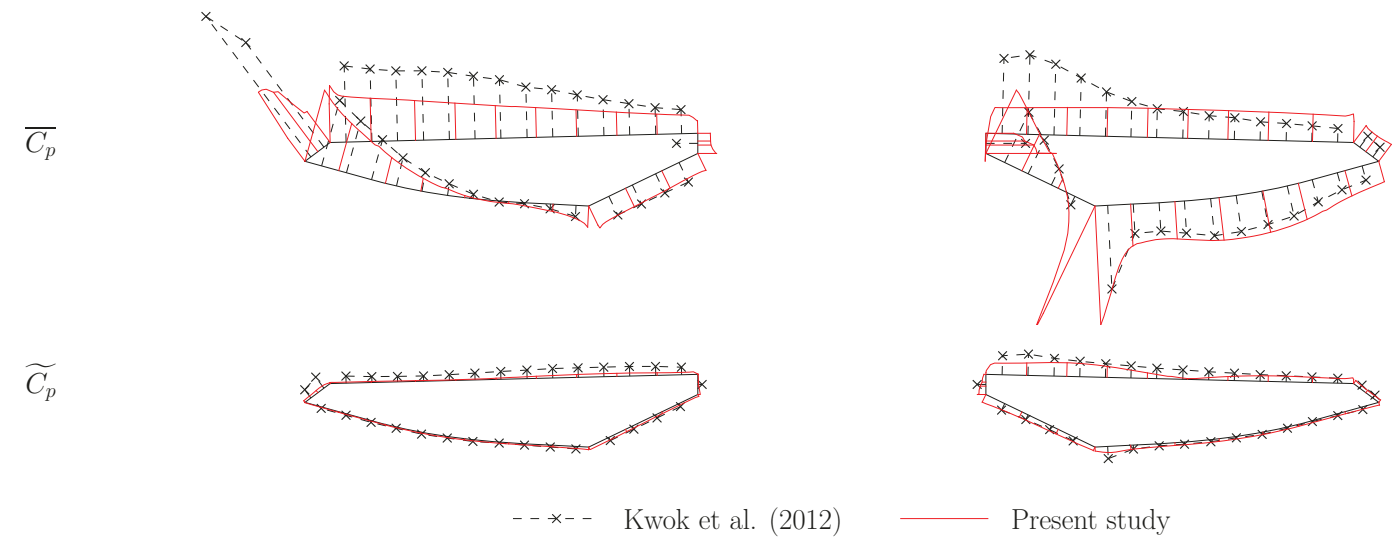

Figure 17: $\overline{C_{p}}$ and $\widetilde{C_{p}}$ distributions for Gap K and Gap04 at $10^{\circ}$ angle of attack. (Negative values points outward the section and the height of the section is equal to a value of unity.) 


\section{$5.2 \quad$ Force coefficients}

In figures 18 to 20 the force coefficients in the ranges of angles of attack $\left(-10^{\circ},+10^{\circ}\right)$ for the fourteen different gap widths considered in this study are presented. Along with the CFD-based results, the wind tunnel values reported in Kwok et al. (2012) for five of the considered geometries are also included for validation. The reference dimension considered for the force coefficients (equation (4)) is the width of one box $(C)$ ). These results show a complete panorama in terms of the expected aerodynamic performance of the deck cross-section as the gap distance between boxes is modified.

For the drag coefficient, the investigated arrangements show a roughly parabolic trend, which is typical of streamlined box decks. Also, for $G / D$ ratios higher than 1.5 (Gap $\mathrm{G}$ and successive) the $\overline{C_{d}}$ curve is shifted upwards providing higher values for the drag coefficient. This is due to the increment in the positive mean pressure acting on the panels facing the gap in the downwind box as the gap distance increases. The accuracy of the CFD simulations can be judged as fairly good when experimental and numerical values are compared. The CFD-based simulations reproduce faithfully the trend in the drag coefficients and discrepancies in the values for individual arrangements and angles of attack are worthy of mention only in a limited number of realisations such as Gap B at $\alpha=-1^{\circ}$, Gap H at $\alpha=5^{\circ}$ or Gap K at $\alpha=7^{\circ}$. In these cases, the 2D URANS simulations are providing a steady solution, which might explain the differences with the wind tunnel results.

The lift coefficient shows a linear trend in the central part of the studied range of angles of attack, which is the expected trend for streamlined bridge decks. The slope of the lift coefficient is positive, which is indicative of an aeroelastic performance not prone to suffer from flutter instability (Larose et al., 1997; Diana et al., 2007). The range of values obtained for the $C_{l}$ do not change significatively for the studied gaps. When the numerical results are compared with the available wind tunnel data, the agreement is remarkably good, particularly in terms of the lift coefficient slope. Some differences can be appreciated only in the higher part of the angles of attack range $\left(5^{\circ}, 7^{\circ}\right.$ and $\left.10^{\circ}\right)$ on Gaps $\mathrm{H}, \mathrm{K}$ and $\mathrm{L}$, as massive flow separation takes place and the CFD simulations are not able to accurately reproduce the strong suctions along the top surfaces of the deck, as it was previously explained for the Gap $\mathrm{K} \alpha=10^{\circ}$ case.

The moment coefficient shows also a clear linear trend in the central range of angles of attack considered for the studied deck arrangements. The moment coefficients behave in a similar manner as the lift coefficient, that is, the slope is roughly constant as well as the range of the values of the moment coefficient for the studied deck geometries. The agreement between numerical and wind tunnel data is remarkably good for small gap widths (Gap A and Gap B). Nevertheless, the accuracy deteriorates to some extent as the gap widens due to the effect caused by the increase in the distance from the reference axis to the centre of pressures of each box, as has been previously explained in section 4. In any case, these discrepancies do not compromise the ability of the CFD simulations to provide meaningful insights into the moment coefficient values for the considered twin-box arrangements. 

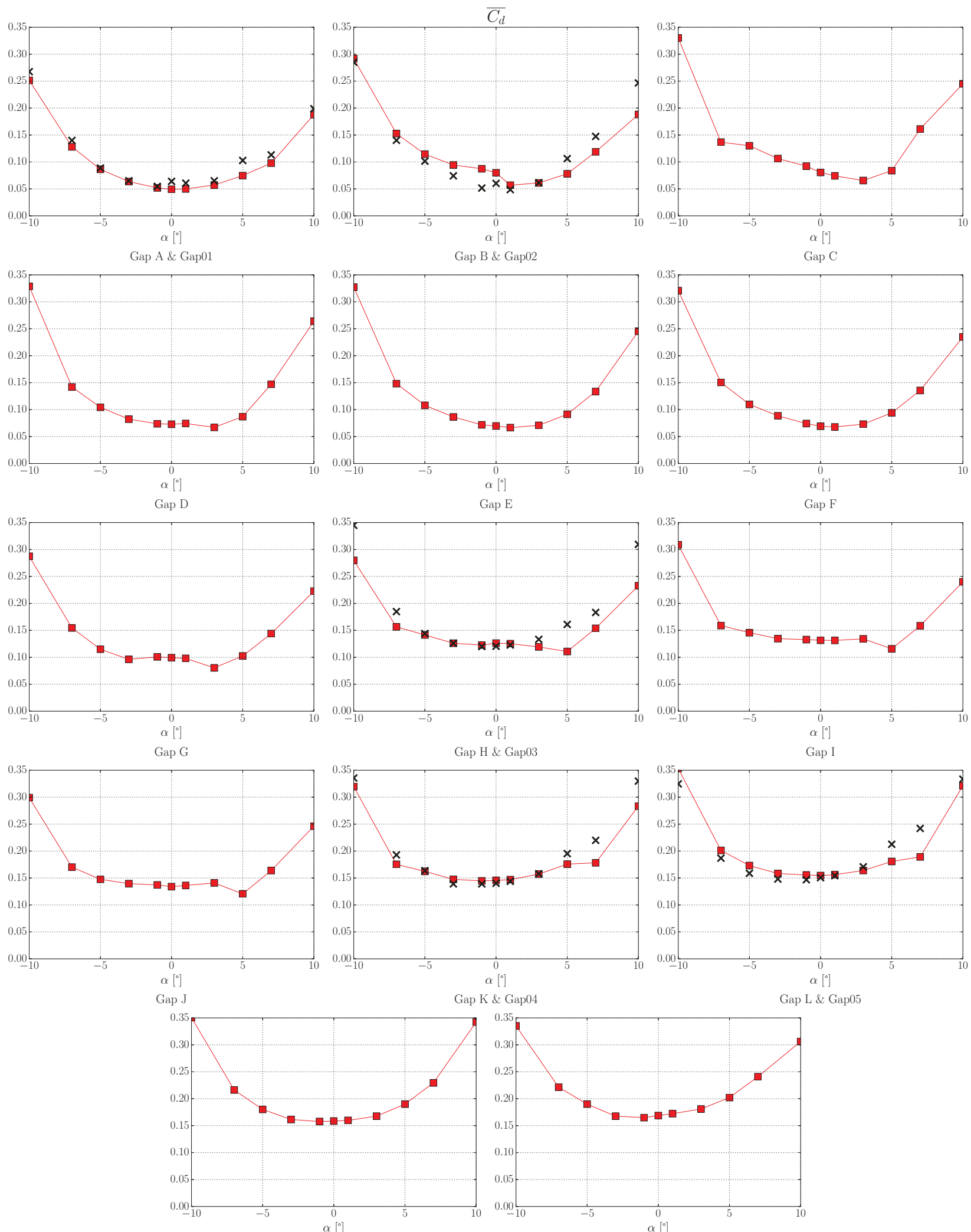

Gap M

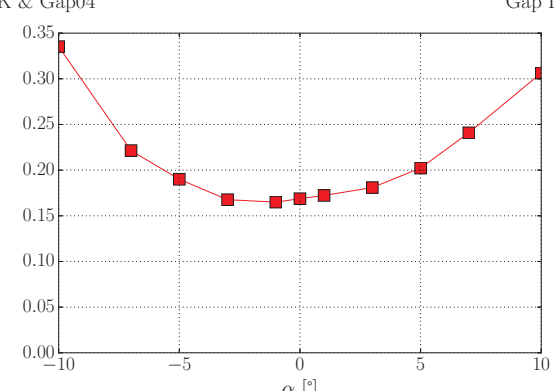

- Present study $\quad$ Kwok et al. (2012)

Figure 18: Mean drag coefficient $\left(\overline{C_{d}}\right)$ for different gap widths and angles of attack. 

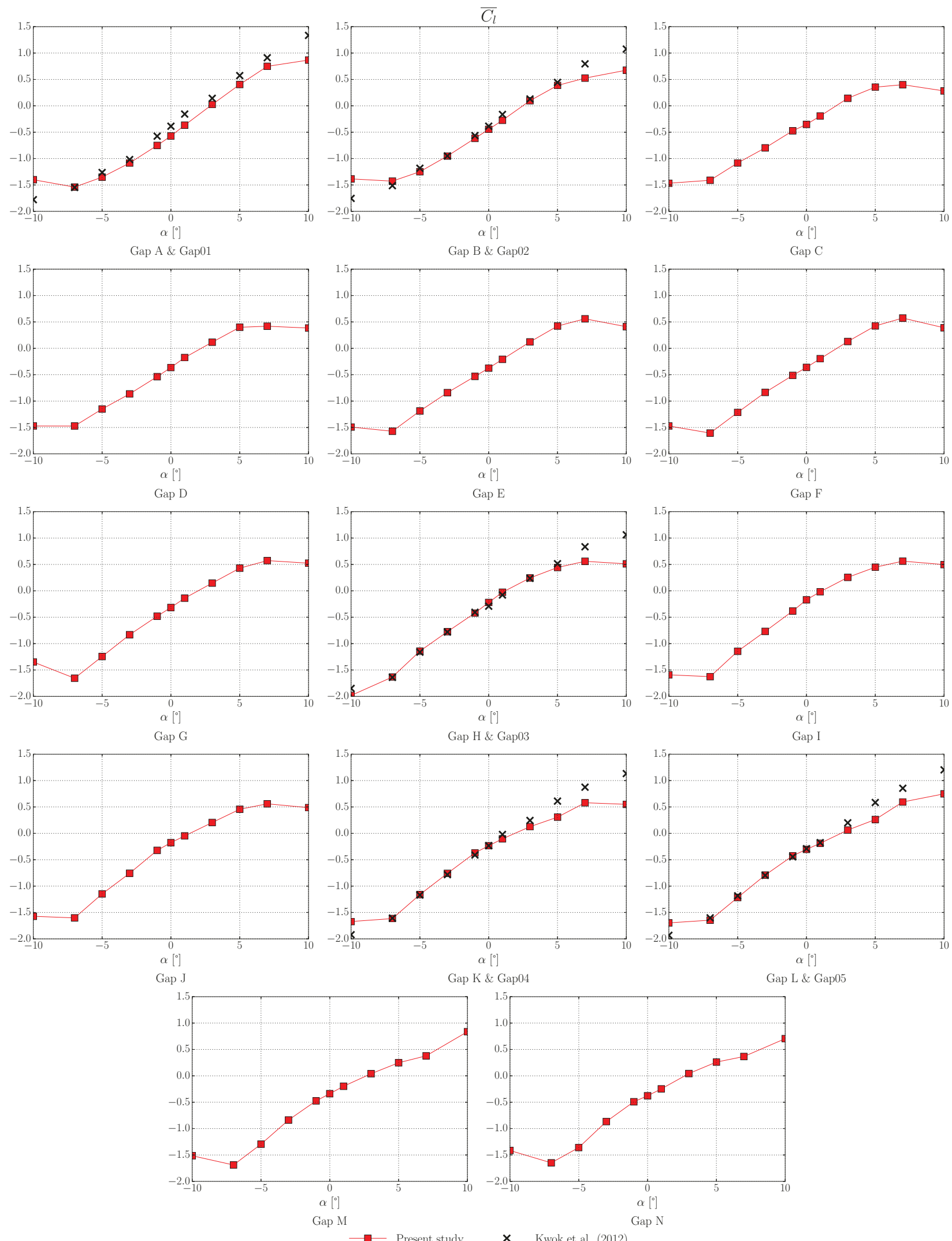

- Present study $\quad$ Kwok et al. (2012)

Figure 19: Mean lift coefficient $\left(\overline{C_{l}}\right)$ for different gap widths and angles of attack. 

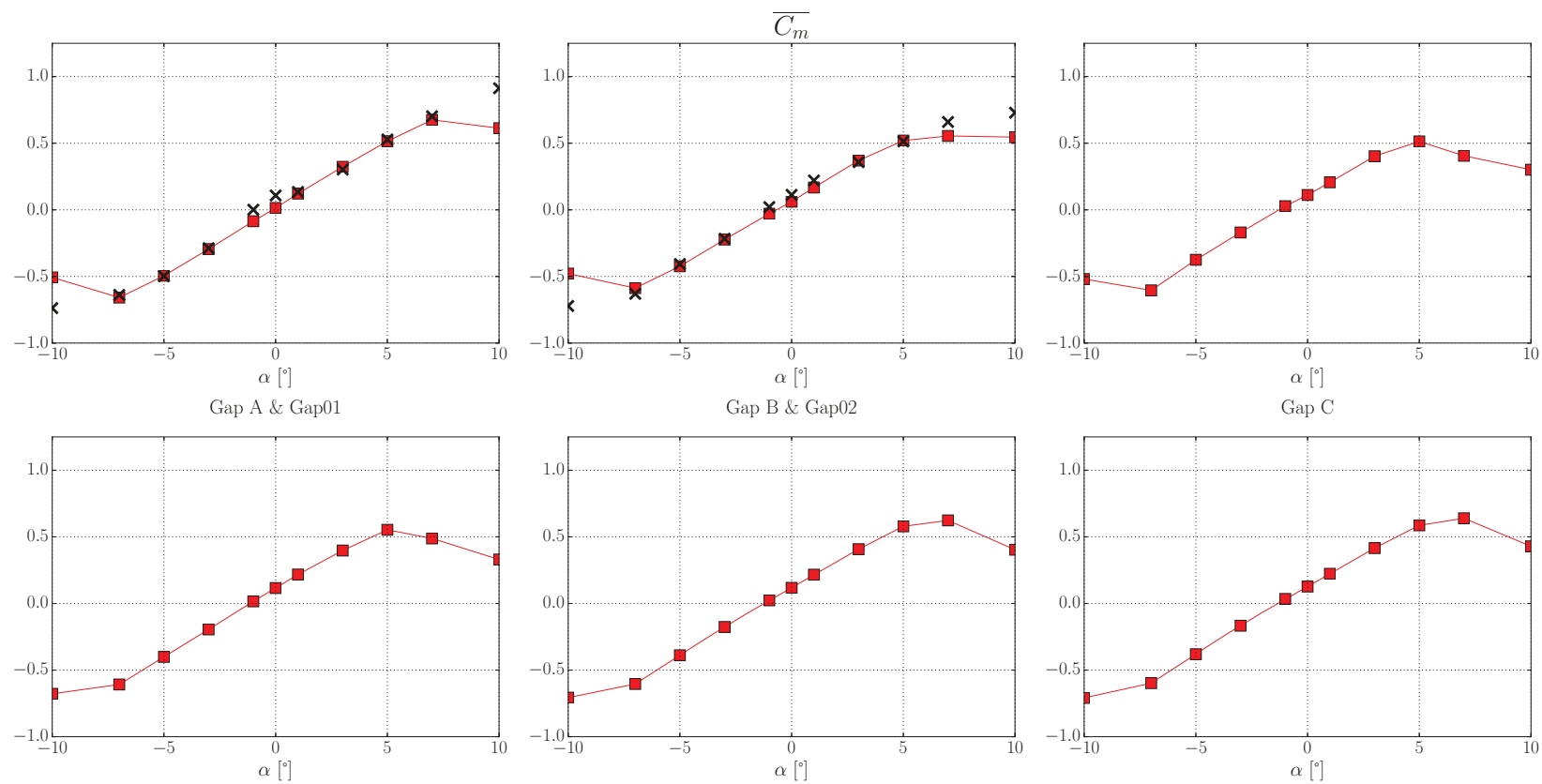

Gap B \& Gap02

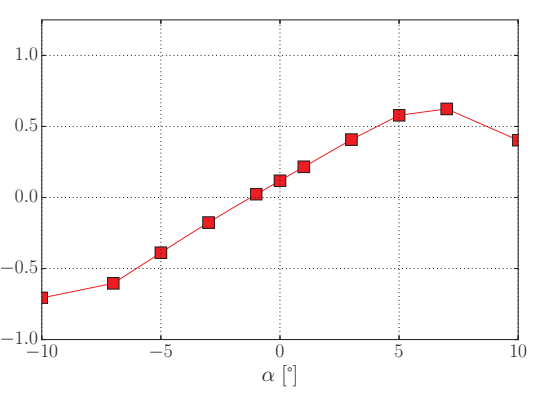

Gap C
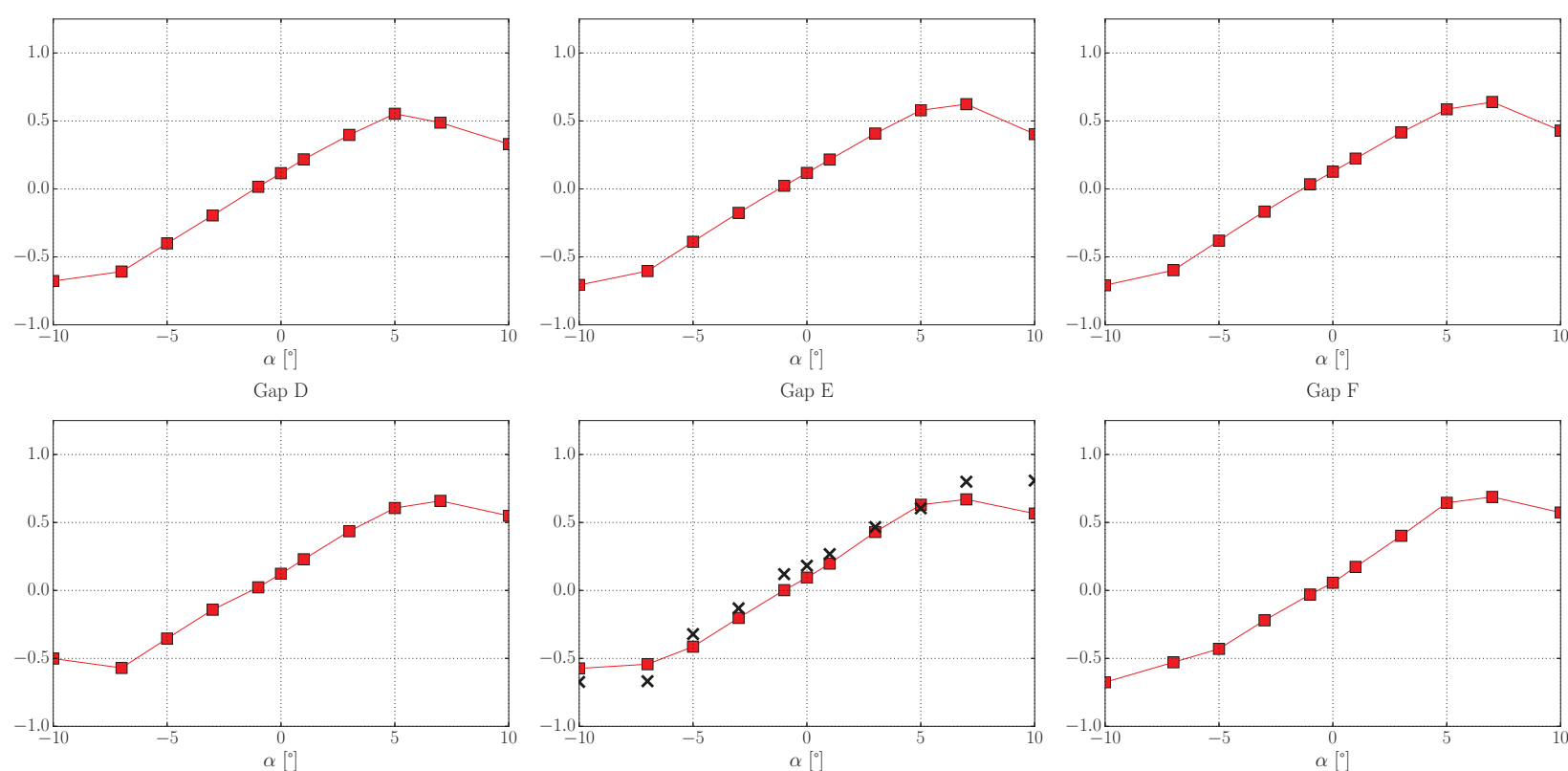

Gap G

Gap H \& Gap03
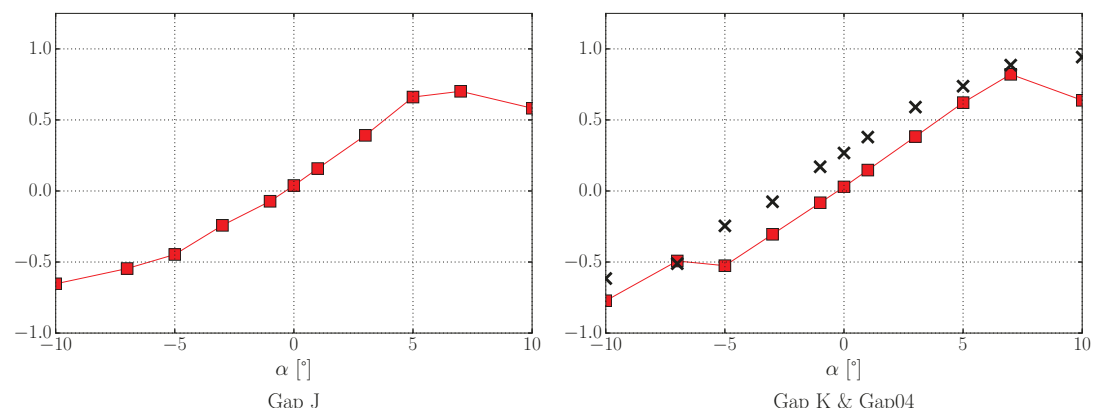

Gap K \& Gap04

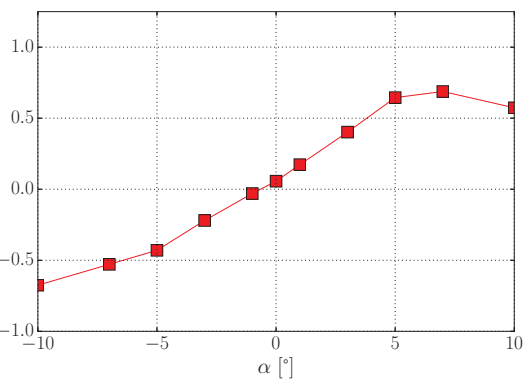

Gap I
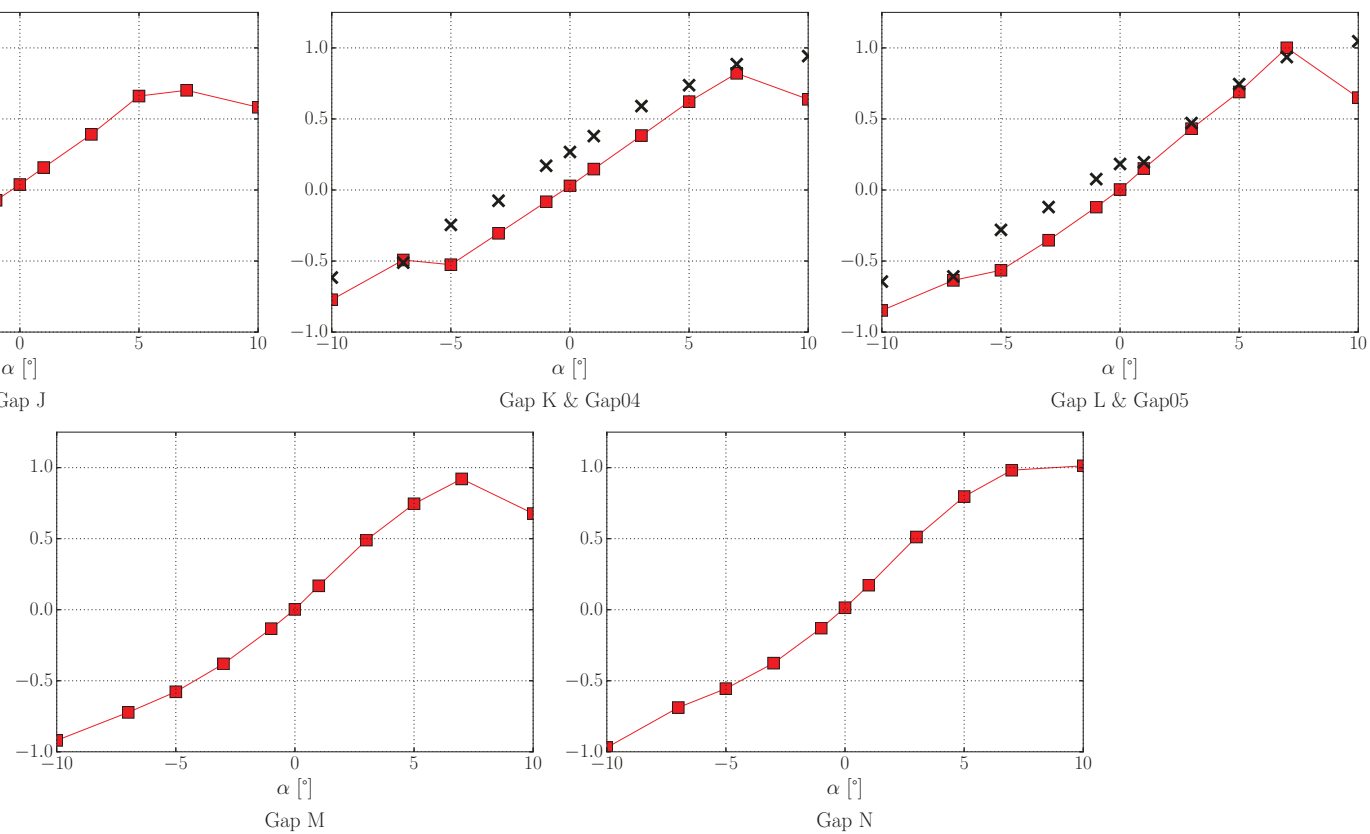

Gap N

Figure 20: Mean moment coefficient $\left(\overline{C_{m}}\right)$ for different gap widths and angles of attack. 


\subsection{Vortex shedding mechanism}

The snapshots of the instantaneous vorticity fields that for different gap to depth ratios provided allowed to identify the existence of vortex shedding and its types: Patterns A or B, according to Laima et al. (2015), or first, second or third sources, according to Kwok et al. (2012). In Pattern A, vortices are shed from the leeward edges of the downwind box, on the other hand, Pattern B is characterised by the existence of vortex shedding detaching from the leeward edges of the upwind box. These vortices drift along the gap impinging on the downwind box. Kwok et al. (2012) described the first source of vortex shedding as the vortices shed from the upwind deck and the second source as the vortices shed from the downwind box. The third source is characterised by the turbulence buffeting excitation of the downwind box due to the impingement of individual vortices coming from the upwind box. Pattern A in Laima et al. (2015) is equivalent to source 2 in Kwok et al. (2012), while Pattern B may be considered as equivalent to the combination of source 1 and source 3 as discussed in Kwok et al. (2012).

The data provided by the numerical simulations may further help to identify the type of vortex shedding that is taking place for a certain gap ratio. In the following, for Gap K, pressure spectra at certain locations are studied and the results compared with the ones reported in Kwok et al. (2012).

Vortex shedding may induce the oscillatory motion of a bridge deck. Experimental VIV tests reported in Laima et al. (2015) have identified different types of vortex-induced vibrations depending on the gap ratio: vertical VIV, both vertical and torsional VIV and only torsional VIV. Aiming to provide a preliminary assessment of the VIV susceptibility and type for the studied deck geometries, the phase lags between instantaneous force coefficients acting on each individual box and the standard deviation of the total lift and moment coefficients are also investigated.

\subsubsection{Pressure spectra}

In order to analyse the susceptibility of the different cross-sections studied to encounter Vortex Induced Vibration (VIV), the spectra of the pressure time-histories at points located at four potential sources of vortex shedding are studied. These locations are the trailing edges of the upwind box and the windward faces of the downwind box (Kwok et al., 2012). In figure 21, the pressure spectra for the different locations on the Gap K geometry, considered as representative of large gap cases, are shown. The spectra peaks that can be seen in figure 21 are coincident with the $S t$ obtained from the analysis of the lift time-history and they are in very good agreement with the experimental results reported in Kwok et al. (2012). The differences in the amplitudes between CFD and wind tunnel data that can be appreciated in figure 21 for non-dimensional frequencies different from the Strouhal number and its multiple are due to the inability of URANS models to resolve the turbulence scales, producing single mode large scale structures.

In the rest of configurations studied, the same behaviour is appreciated, that is, the pressure spectra peaks are associated with the same value of the $S t$.

Therefore these CFD simulations have been able to accurately replicate the vortex shedding mechanism associated with the "first source" and "third source" types discussed in Kwok et al. (2012). 


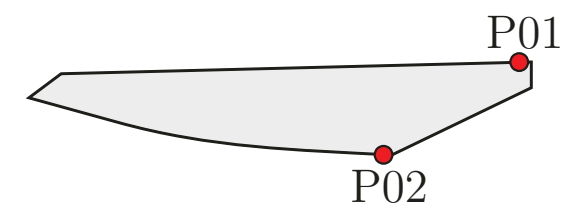

(a)
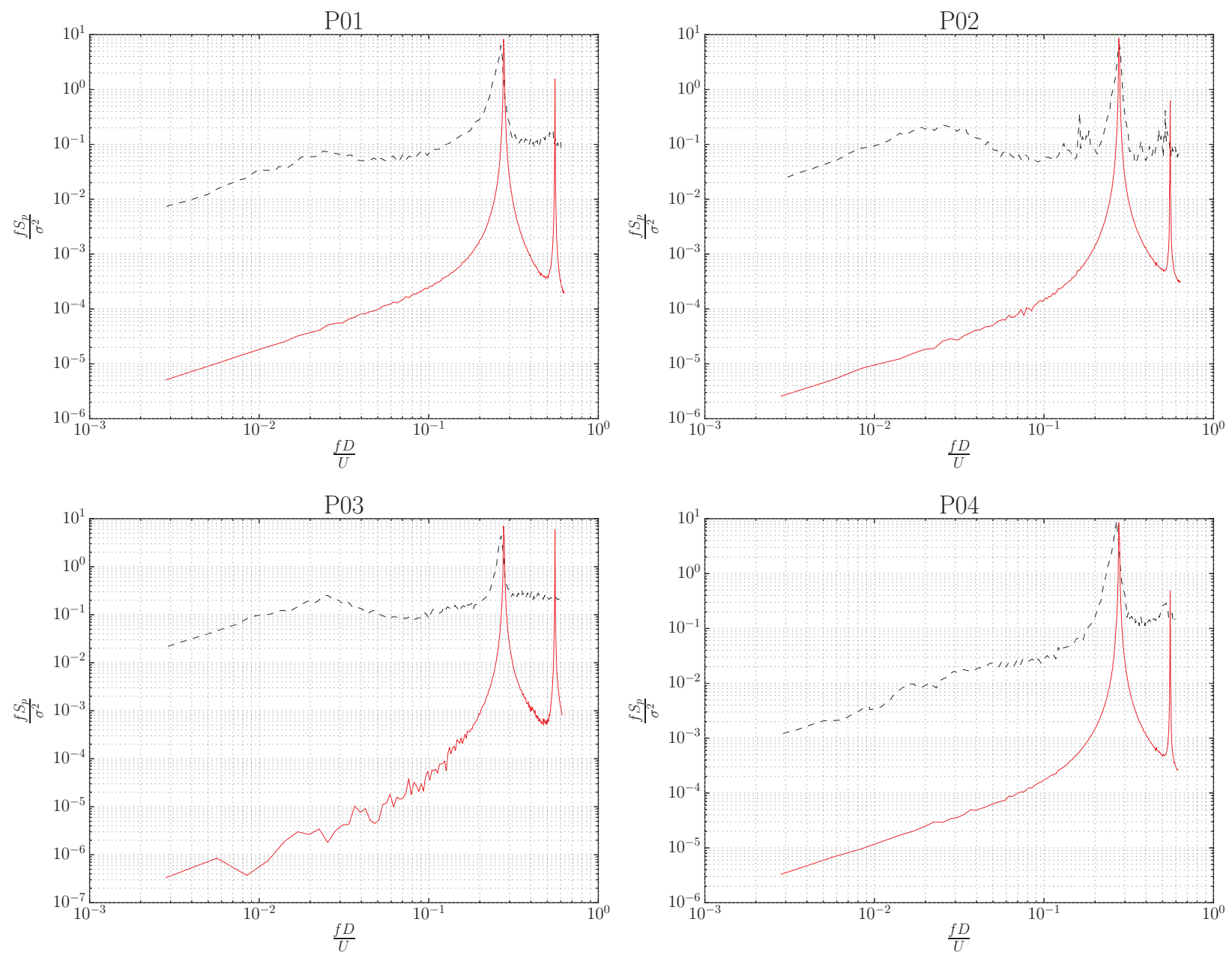

Kwok et al. (2012)
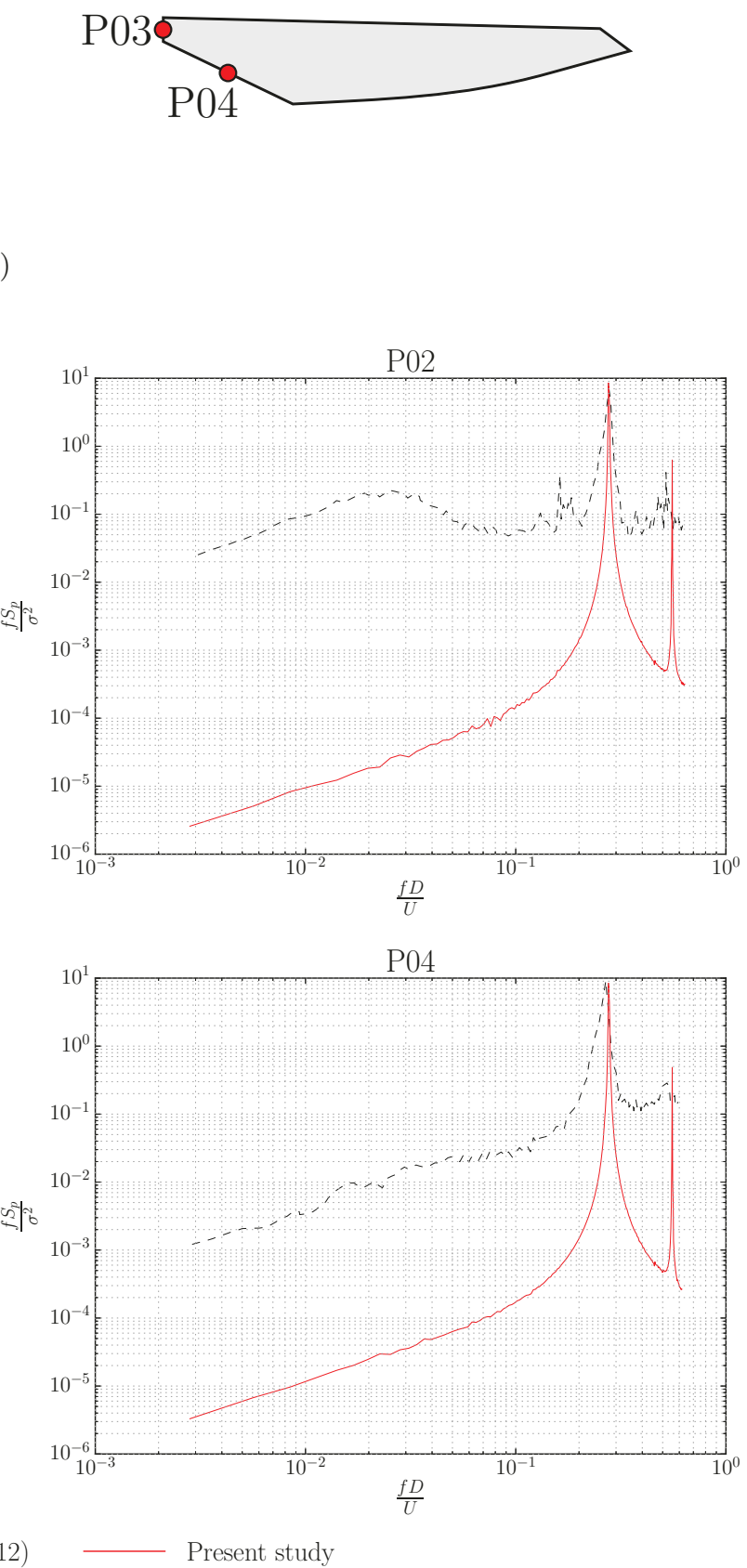

(b)

Figure 21: a) Measurement points locations and b) pressure spectra at different locations for the Gap K at $0^{\circ}$ angle of attack. 


\subsubsection{Force coefficients, phase lags and standard deviations}

The phase lags between the force coefficients time-histories acting on the two individual boxes are presented in figure 22 for the different gap widths at $0^{\circ}$ angle of attack. The time histories for each gap width computed for each box, have the same predominant frequency as the one reported in the calculation of the Strouhal number for the overall cross-section as it was demonstrated studying the pressure spectra. The phase lag between the lift time-histories was calculated via the Fourier transforms of the lift coefficients time-histories acting on each box. Then the phase lag is identified for the dominant frequency in the time-histories. The investigation of the phase lags can provide an idea about the susceptibility of each arrangement to suffer vortex induced vibration and the type of VIV mechanism. If the phase lag between the lift forces acting on each individual box is almost null, or close to $360^{\circ}$, these instantaneous lift forces are acting in the same direction on both boxes at each instant, which can enhance the amplitude in the lift coefficient. On the other hand, when the phase lags between the lift forces on each box are close to $180^{\circ}$, they are acting on both boxes in opposite directions every time, which enhances the moment amplitude, increasing rotational vibrations. In figure 23 the phenomenon previously explained is shown, where the gaps with a phase lag from the $C_{l}$ time-histories closer to $0^{\circ}$ or $360^{\circ}$, such as Gap $\mathrm{G}(G / D=1.66)$ and $\mathrm{K}(G / D=3.96)$, have a greater $\widetilde{C_{l}}$ value due to the summation of the contribution of both boxes and a smaller overall $\widetilde{C_{m}}$. Meanwhile the cases with phase lags closer to $180^{\circ}$ from the $C_{l}$ time-histories, such as Gap L $(G / D=5.85)$, present a higher overall $C_{m}$ and smaller overall $\widetilde{C_{l}}$. In some cases, such as Gap H, I and $\mathrm{J}(2.08 \leq G / D \leq 2.63)$, relatively high values for both the $\widetilde{C_{l}}$ and the $\widetilde{C_{m}}$ are obtained, which may be potentially indicative of simultaneous vertical and torsional VIV.
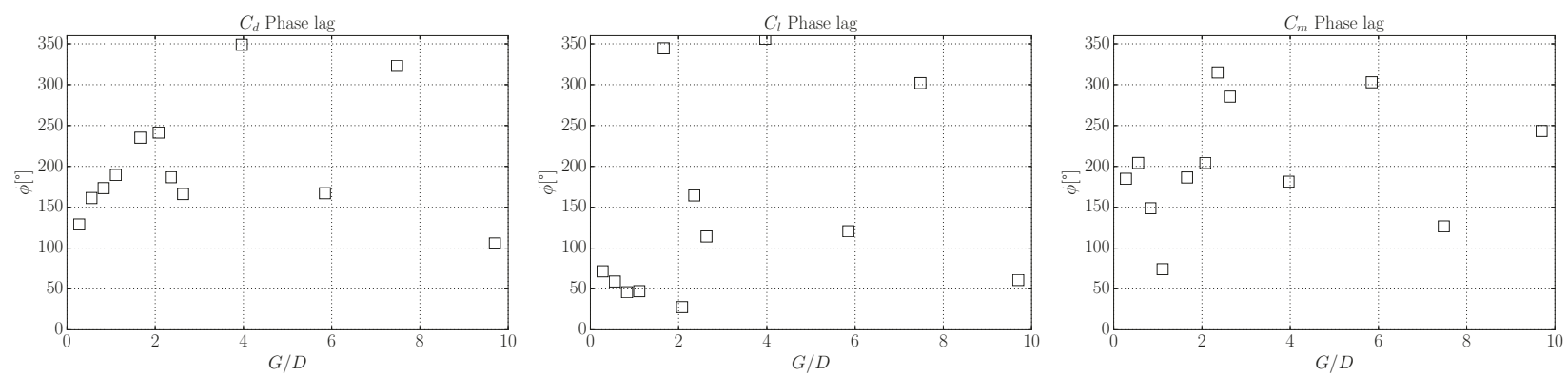

Figure 22: Phase lags between the boxes force coefficients for different gap widths at $0^{\circ}$ angle of attack.
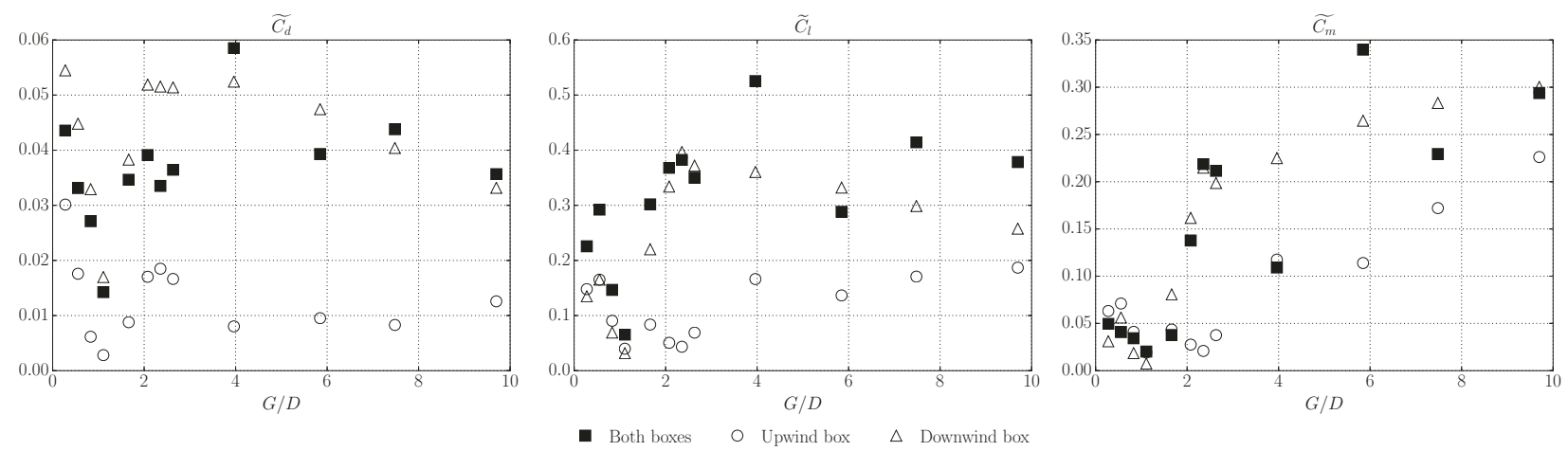

Figure 23: Standard deviations for different gap widths at $0^{\circ}$ angle of attack. 


\subsection{Critical gap ratio identification}

Experimental studies have allowed the identification of the so-called critical gap ratio for twin-box decks, for which the flow motion in the gap suddenly changes (Laima et al., 2015). In the aforementioned reference, the value provided as critical gap ratio for a twin-box deck with the geometry of the Xihoumen Bridge was 2.138. In previous sections, the snapshots of instantaneous vorticity fields have allowed to identify different flow features for small and large gap ratios, identifying the vortex shedding patters A and B, described in the aforementioned reference. In this section, the force coefficients at $0^{\circ}$ angle of attack and the Strouhal number are analysed aiming to identify the critical gap ratio for the Stonecutters Bridge deck geometry and further contribute to the assessment of the generality of the critical gap ratio concept.

\subsubsection{Force coefficients vs. gap ratio}

The force coefficients values obtained from the numerical simulations conducted for the different gaps at zero degrees of angle of attack are presented in figure 24 . Referring to the $\overline{C_{d}}$, its value is kept nearly constant, in the range $0.28 \leq G / D \leq 1.38$. Once the gap width is big enough to allow the vortices shed from the upwind box to develop and impinge upon the downwind one, the drag coefficient increases more quickly up to $G / D=2.08$ and more slowly afterwards. On the other hand, the $\overline{C_{l}}$ presents, in general, an increment in value up to $G / D=2.35$, subdivided in two ramps with an intermediate plateau. In the first ramp, $G / D \leq 0.55$, the increment in value is due to the increment of the suction on the upper surfaces of both boxes in the vicinity of the gap. Between the two ramps, it exists a plateau where the value of $\overline{C_{l}}$ slightly decreases $(0.55<G / D<1.66)$, as the suction over the inclined panel of the downwind box increases and the suctions over the upper panel of the same box, diminish. Finally, the second ramp of $\overline{C_{l}}$ growth $(1.66 \leq G / D \leq 2.35)$, is basically due to the presence of positive pressures on the inclined panel of the downwind box as the vortices shed from the upwind box have more room to develop and impinge on the downwind box. From $G / D>2.35$ the value of $\overline{C_{l}}$ decreases steadily as the suction values on the upper surface of the downwind deck decrease, because, the greater the gap the weaker the vortices impinge upon the downwind box, as the vortices have been able to fully develop and then slowly dissipate as the gap distance to travel increases. The moment coefficient shows a step growth for $G / D \leq 0.55$, as it happened with the lift coefficient. Then, a plateau can be identified in the range $0.55<G / D<1.66$, followed by a step decrement up to $G / D=2.35$, obtaining almost null values for the moment coefficient for $G / D>2.35$, once the gap distance is enough for the full development of the vortices shed from the upwind box. According to the comments above, the critical gap ratio can be identified for $G / D=2.35$.

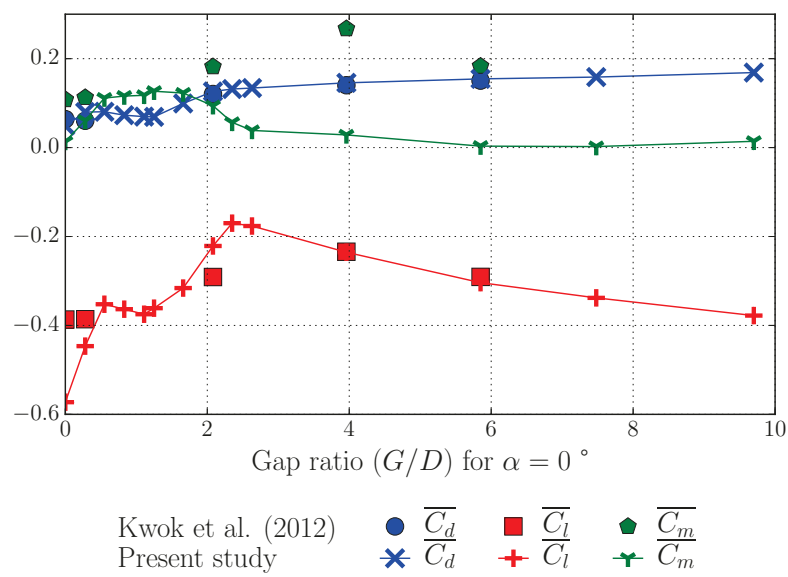

Figure 24: Force coefficients for the different gap widths at $\alpha=0^{\circ}$. 


\subsubsection{Strouhal number vs. gap ratio}

The values of the Strouhal number for each gap to depth ratio at an angle of attach of $0^{\circ}$ are presented in figure 25. In general, the greater the gap width, the greater the Strouhal number value. Also two regions with different growth trends in the values can be identified. The first of them presents a linear trend with steeper slope until reaching Gap I $(G / D=2.35)$. This first region corresponds to gap widths in which the vortices shed from the upwind box are not fully developed, as it was previously commented upon, and there is a weak vortex in the wake of the downwind box. On the other hand, the second region presents a smoother increment in the value of $S t$, which tends towards an asymptotic value for the greater gap widths. This second region would be related with the gap widths in which the vortices are fully developed. These two regions are also identified in the work of Laima et al. (2015), although for a different twin-box deck section geometry, which shows the generality of the $S t$ dependency as a function of the gap width.

It must be remarked that the numerical values of the Strouhal number are in very good agreement with the experimental ones. It must be noted that the $S t$ values in the numerical simulations are calculated adopting as reference dimension the value for the deck depth $D=3.609 \mathrm{~m}$ (Larose et al., 2003) instead of $D=3.5 \mathrm{~m}$ used by Kwok et al. (2012). Therefore the experimental values in Kwok et al. (2012) have been recalculated using $D=3.609 \mathrm{~m}$ in order to compare the experimental and numerical results.

A fitting curve was approximated for the numerical results in each identified region. The equations of the two fitting curves are presented next, which correspond to a linear fitting in Region 1 and an exponential one in Region 2, in agreement with Laima et al. (2015).

$$
\begin{aligned}
& \text { Region 1: } S t=0.071+0.0744 \frac{G}{D} \\
& \text { Region 2: } S t=0.318-0.2505\left(\frac{G}{D}\right)^{-1.3362}
\end{aligned}
$$

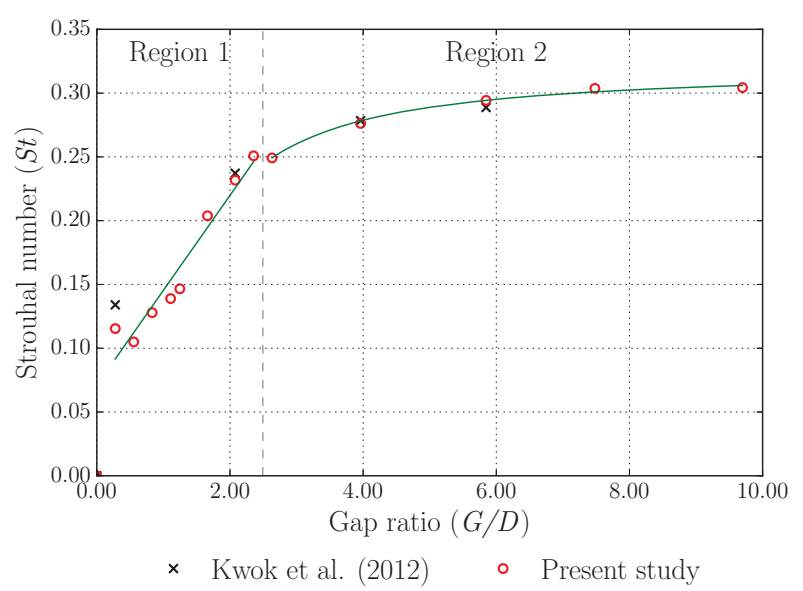

Figure 25: Strouhal number for the different gap widths at $\alpha=0^{\circ}$.

The critical gap ratio for the Stonecutters Bridge deck geometry identified based on CFD simulations is $G / D=2.35$, which is consistent with the value obtained from the study of the force coefficients, and in close agreement with the value of $G / D=2.135$ proposed by Laima et al. (2015) for the Xihoumen Bridge deck geometry. 


\section{Conclusions}

Relatively inexpensive 2D URANS simulations have been adopted to study the aerodynamic response in smooth flow of a twin-box bridge deck. Fourteen different gap to depth ratios have been investigated $(0 \leq G / D \leq 9.7)$, considering eleven angles of attack in the range $\left(-10^{\circ}, 10^{\circ}\right)$.

Verification studies have allowed the identification of the spatial and time discretisations that provide grid resolution and time step independent results, with lower computational costs. The cells count is about one order of magnitude higher than in equivalent studies (de Miranda et al., 2015) however, the agreement between numerical and experimental results is remarkably improved.

The snapshots of the instantaneous vorticity fields have allowed identifying the fundamental flow features for different values of the gap to depth ratios. These data, in combination with the mean and fluctuating pressure coefficients, the pressure spectra and phase lags between the force coefficients of each box have permitted the understanding of the different vortex shedding mechanisms, which agree with the proposals based on wind tunnel tests reported by Kwok et al. (2012) and Laima et al. (2015). The agreement in the mean pressure coefficients distributions between the numerical simulations and the wind tunnel tests is very good for the studied range of gap to depth ratios. Only in the higher range of angles of attack $\left(7^{\circ}, 10^{\circ}\right)$ non-negligible differences are apparent over the top surfaces of the boxes, and a plausible explanation is provided based on the known limitations of two-equation turbulence models. In the same manner, the numerical simulations reported herein typically overestimate the standard deviation of the pressure coefficients in the regions where vortices detach or impinge, which is characteristic of the adopted $k-\omega$ SST turbulence model.

The global aerodynamic behaviour of the studied twin box deck as a function of the gap ratio and the angle of attack is assessed by means of the force coefficients. The general agreement with the wind tunnel results is very good. Apart from the differences already mentioned for high positive angles of attack, some discrepancies can be appreciated in the moment coefficients for large gap ratios due to the multiplicative effect caused by the lever arm over the differences in the mean pressure distributions. The critical gap ratio for the studied twin box deck has been identified and the result is consistent with previous experimental studies conducted for the Xihoumen Bridge (Laima et al., 2015). The values obtained for the Strouhal number for different gaps are in very good agreement with experimental data and the trend in the Strouhal number as a function of the gap to depth ratio is coincident with the experimental data reported in the literature.

This work has shown the potential of 2D URANS simulations to provide reliable information about the complex aerodynamic behaviour of long-span bridge decks at a moderate computational and budgetary cost. This is judged of utmost importance at the early design stage in industrial projects of cable-supported bridges.

\section{Acknowledgements}

This research has been funded by the Spanish Ministry of Economy and Competitiveness in the frame of the research project with reference BIA2016-76656-R and the Galician regional goverment with reference ED431C 2017/72. The first of the authors has been funded by the Spanish Ministry of Economy and Competitiveness in the frame of the National Program for Promotion of Talent and Employability through the BES-2014-068418 predoctoral contract grant, associated with the BIA2013-41965-P research project.

The computations have been carried out in the computer cluster Breogan and in the Galician Supercomputation Technology Centre (CESGA).

The authors fully acknowledge the received support. 


\section{References}

Bruno, L., D. Fransos, N. Coste, and A. Bosco (2010). "3D flow around a rectangular cylinder: A computational study". In: Journal of Wind Engineering and Industrial Aerodynamics 98, pp. 263-276.

Chen, W. L., H. Li, and H. Hu (2014). "An experimental study on the unsteady vortices and turbulent flow structures around twin-box-girder bridge deck models with different gap ratios". In: Journal of Wind Engineering and Industrial Aerodynamics 132, pp. 27-36.

Collie, S., M. Gerritsen, and P. Jackson (2008). "Performance of two-equation turbulence models for flat plate flows with leading edge bubbles". In: Journal of Fluids Engineering 130.

de Miranda, S., L. Patruno, M. Ricci, and F. Ubertini (2015). "Numerical study of a twin box bridge deck with increasing gap ratio by using RANS and LES approaches". In: Engineering Structures 99, pp. 546-558.

Diana, G., M. Belloli, F. Resta, and A. Zasso (2007). "Sensitivity analysis on the effects of different aerodynamic devices on the behavior of a bridge deck". In: Proceedings of the 12 th International Conference on Wind Engineering, Cairns, Australia.

Dragomirescu, E., Z. Wang, and M. S. Hoftyzer (2016). "Aerodynamic characteristics investigation of Megane multi-box bridge deck by CFD-LES simulations and experimental tests". In: Wind and Structures An International Journal 22.2, pp. 161-184.

Hui, M. C. H., Z. Y. Zhou, A. R. Chen, and H. F. Xiang (2008). "The effect of Reynolds numbers on the steady state aerodynamic force coefficients of the Stonecutters bridge deck section". In: Wind and Structures 3, pp. $179-192$.

Kwok, K. C. S., X. R. Qin, C. H. Fok, and P. A. Hitchcock (2012). "Wind-induced pressures around a sectional twin-deck bridge model: Effects of gap-width on the aerodynamic forces and vortex shedding mechanisms". In: Journal of Wind Engineering and Industrial Aerodynamics 110, pp. 50-61.

Laima, S. and H. Li (2015). "Effects of gap width on flow motions around twin-box girders and vortex-induced vibrations". In: Journal of Wind Engineering and Industrial Aerodynamics 139, pp. 37-49.

Laima, S., H. Li, W. Chen, and F. Li (2013). "Investigation and control of vortex-induced vibration of twin box girders". In: Journal of Fluids and Structures 39, pp. 205-221.

Langtry, R. B. and F. R. Menter (2009). "Correlation-based transition modeling for unstructured parallelized computational fluid dynamics codes". In: AIAA Journal 47.12, pp. 2894-2906.

Larose, G. L., S. V. Larsen, A. Larsen, M. Hui, and A. G. Jensen (2003). "Sectional model experiments at high Reynolds number for the deck of a $1018 \mathrm{~m}$ span cable-stayed bridge". In: Proceedings of The Eleventh International Conference on Wind Engineering. Lubbock, Texas, USA.

Larose, G. L. and F. M. Livesey (1997). "Performance of streamlined bridge decks in relation to the aerodynamics of a flat plate". In: Journal of Wind Engineering and Industrial Aerodynamics 89, pp. 1591-1606.

Larsen, A. and S. Poulin (2005). "Vortex-shedding excitation of box-girder bridges and mitigation". In: Journal of the International Association for Bridge and Structural Engineering (IABSE) 15.4, pp. 258-263.

Larsen, A., M. Savage, A. Lafreniere, M. C. H. Hui, and S. V. Larsen (2008). "Investigation of vortex response of a twin box bridge section at high and low Reynolds numbers". In: Journal of Wind Engineering and Industrial Aerodynamics 96, pp. 934-944.

Lee, S., S. Kwon, and J. Yoon (2014). "Reynolds number sensitivity to aerodynamic forces of twin box bridge girder". In: Journal of Wind Engineering and Industrial Aerodynamics 127, pp. 59-68.

Li, H., S. Laima, and H. Jing (2014). "Reynolds number effects on aerodynamic characteristics and vortexinduced vibration of a twin-box girder". In: Journal of Fluids and Structures 50, pp. 358-375.

Mannini, C., A. Soda, and G. Schewe (2010). "Unsteady RANS modelling of flow past a rectangular cylinder: Investigation of Reynolds number effects". In: Computers $\&$ Fluids 39, pp. 1609-1624.

Menter, F. and T. Esch (2001). "Elements of industrial heat yransfer prediction". In: Proceedings of the 16th Brazilian Congress of Mechanical Engineering. Vol. 20, pp. 117-127.

Menter, F. R. (2009). "Review of the shear-stress transport turbulence model experience from an industrial perspective". In: International Journal of Computational Fluid Dynamics 23.4, pp. 305-316.

Nieto, F., I Kusano, S. Hernández, and J. Á. Jurado (2010). "CFD analysis of the vortex-shedding response of a twin-box deck cable-stayed bridge". In: Proceedings of The Fifth International Symposium on Computational Wind Engineering (CWE2010). Chapel Hill, USA.

Nieto, F., J. S. Owen, D. M. Hargreaves, and S. Hernandez (2015). "Bridge deck flutter derivatives: Efficient numerical evaluation exploiting their interdependence". In: Journal of Wind Engineering and Industrial Aerodynamics 136, pp. 138-150. 
Nieto, F., A. Zasso, D. Rocchi, and S. Hernández (July 2008). "CFD verification of aerodynamic devices performance for the MESSINA Strait Bridge". In: Proceedings of the VI International Colloquium on: Bluff Bodies Aerodynamics 83 Applications. Milano, Italy, pp. 1-9.

Ozkan, E., D. Rocchi, A. Allsop, S. Kite, and A. Zasso (2011). "The aerodynamic performance of Forth Replacement Crossing". In: Proceedings of the Thirteenth International Conference on Wind Engineering. Amsterdam, The Netherlands.

Patruno, L. (2015). "Accuracy of numerically evaluated flutter derivatives of bridge deck sections using RANS: Effects on the flutter onset velocity". In: Engineering Structures 89, pp. 49-65.

Qin, X. R., K. C. S. Kwok, C. H. Fok, P. A. Hitchcock, and X. L. Xu (2007). "Wind-induced self-excited vibrations of a twin-deck bridge and the effects of gap-width". In: Wind and Structures, An International Journal 10.4, pp. 37-49.

Ricciardelli, F. and H. Hangan (2001). "Pressure distribution and aerodynamic forces on stationary box bridge sections". In: Wind and Structures 4.5, pp. 399-412.

Sarkic, A., R. Fisch, R. Hoffer, and K. Bletzinger (2012). "Bridge flutter derivatives based on computed, validated pressure fields". In: Journal of Wind Engineering and Industrial Aerodynamics 104-106, pp. 141-151.

Wilcox, D. C. (2006). Turbulence modelling for CFD. 3rd ed. La Cañada: DCW Industries.

Yang, Y., T. Wu, Y. Ge, and A. Kareem (2015). "Aerodynamic stabilization mechanism of a twin box girder with various slot widths". In: Journal of Bridge Engineering 3.20. 